\title{
Higher-order spin-hole correlations around a localized charge impurity
}

\author{
Yao Wang $\odot,{ }^{1, *}$ Annabelle Bohrdt, ${ }^{2,3,4,5}$ Shuhan Ding, ${ }^{1,6,7}$ Joannis Koepsell, ${ }^{8,7}$ Eugene Demler, ${ }^{5,9}$ and Fabian Grusdt ${ }^{6,7, \dagger}$ \\ ${ }^{1}$ Department of Physics and Astronomy, Clemson University, Clemson, South Carolina 29631, USA \\ ${ }^{2}$ Department of Physics and Institute for Advanced Study, Technical University of Munich, 85748 Garching, Germany \\ ${ }^{3}$ Munich Center for Quantum Science and Technology (MCQST), Schellingstrasse 4, 80799 München, Germany \\ ${ }^{4}$ ITAMP, Harvard-Smithsonian Center for Astrophysics, Cambridge, Massachusetts 02138, USA \\ ${ }^{5}$ Department of Physics, Harvard University, Cambridge, Massachusetts 02138, USA \\ ${ }^{6}$ Department of Physics and Arnold Sommerfeld Center for Theoretical Physics (ASC), \\ Ludwig-Maximilians-Universität München, Theresienstrasse 37, München D-80333, Germany \\ ${ }^{7}$ Munich Center for Quantum Science and Technology (MCQST), 80799 München, Germany \\ ${ }^{8}$ Max-Planck-Institut für Quantenoptik, 85748 Garching, Germany \\ ${ }^{9}$ Institute for Theoretical Physics, Wolfgang-Pauli-Str. 27, ETH Zurich, 8093 Zurich, Switzerland
}

(Received 29 December 2020; revised 20 July 2021; accepted 21 July 2021; published 2 September 2021)

\begin{abstract}
Analysis of higher-order correlation functions has become a powerful tool for investigating interacting many-body systems in quantum simulators, such as quantum gas microscopes. Experimental measurements of mixed spin-charge correlation functions in the 2D Hubbard have been used to study equilibrium properties of magnetic polarons and to identify coherent and incoherent regimes of their dynamics. In this paper we consider theoretically an extension of this technique to systems which use a pinning potential to reduce the mobility of a single dopant in the Mott insulating regime of the 2D Hubbard model. We find that localization of the dopant has a dramatic effect on its magnetic dressing. The connected third order spin correlations are weakened in the case of a mobile hole but strengthened near an immobile hole. In the case of the fifth-order correlation function, we find that its bare value has opposite signs in cases of the mobile and of fully pinned dopant, whereas the connected part is similar for both cases. We study suppression of higher-order correlators by thermal fluctuations and demonstrate that they can be observed up to temperatures comparable to the spin-exchange energy $J$. We discuss implications of our results for understanding the interplay of spin and charge in doped Mott insulators.
\end{abstract}

DOI: 10.1103/PhysRevResearch.3.033204

\section{INTRODUCTION}

Understanding strongly correlated systems and their emergent phases of matter has been a central task in modern condensed matter physics. Cuprate superconductors have provided a particularly strong motivation for this line of inquiry because of both potential applications of high-temperature superconductivity and unusual thermodynamic and transport properties of these materials [1]. The single-band (Fermi) Hubbard model has been commonly considered as the minimal microscopic model for describing the physics of high- $T_{c}$ cuprates [2-4]. It correctly reproduces the antiferromagnetic (AFM) Mott insulating state at half filling [5], and is believed to exhibit non-Fermi liquid properties [6-11] as well as $d$ wave pairing at finite doping [12-16], though other lattice and material-specific factors beyond the Hubbard model were

\footnotetext{
*yaowang@g.clemson.edu

${ }^{\dagger}$ Fabian.Grusdt@physik.uni-muenchen.de
}

Published by the American Physical Society under the terms of the Creative Commons Attribution 4.0 International license. Further distribution of this work must maintain attribution to the author(s) and the published article's title, journal citation, and DOI. also argued to be important [17-25]. Methods based on the mean-field approximation are not applicable for analyzing the Hubbard model, because of the presence of many competing instabilities and the importance of quantum antiferromagnetic fluctuations that underlie the emergent nonlocal attraction between electrons [26-29].

Theoretically, the motion of a single hole in a doped Hubbard model takes the form of spin-polaron propagation (sometimes also referred to as magnetic polaron), where the dopant is dressed by a cloud of spin defects in the AFM background [30-40]. Fluctuations of these surrounding spins, correlated with the dopants, should also play a crucial role in the pairing of electrons or holes [41-46]. This mechanism has been supported by multiple solid-state experiments. For example, photoemission (ARPES) experiments have revealed the presence of strong correlations, such as the "high-energy anomaly" and the nonquasiparticle features, even in overdoped cuprates [47-49]; recently resonant inelastic x-ray scattering experiments (RIXS) have also observed the persistent spin fluctuations in a wide range of electron and hole doping [50-54]. Therefore, the correlations between spin and dopants generally exist in strongly correlated materials and may be crucial for the observed emergent phases, including also the spin-glass phase at low doping in disordered cuprate compounds [55]. 
An additional line of inquiry into the nature of strongly correlated electron systems such as cuprates comes from using impurities. One of the most well-studied examples is the nonmagnetic $\mathrm{Zn}$-substitution into the $\mathrm{CuO}_{2}$ plane, which induces charge impurities due to local chemical potential shifts. With this substitution, nuclear magnetic resonance (NMR) and muon spin relaxation ( $\mu$ SR) experiments found that the magnetic moment is locally enhanced near the $\mathrm{Zn}$ impurities [56-59]. At the same time, the substitution heavily suppresses the $d$-wave superconductivity [58-63]. While the mean-field theory can address this pair-breaking phenomenon [64-69], it appears to provide an apparent contradiction to the spin-fluctuation-mediated pairing mechanism: It remains unclear why superconductivity is suppressed while the local moment increases.

As an insightful perspective, we know that it is the dynamical instead of static spin correlations that enhance the $d$-wave superconductivity [70-72]. Therefore, to address this question, one needs to understand the microscopic correlation between the carrier (electron or hole) and the spin fluctuations, and their spatial distribution particularly near the impurity. This is important not only for cuprates but also generally for all correlated materials: As we will show in this paper, a local charge impurity has outsized effects on high-order spin correlations. However, such a correlation involves a dopant hole and electron and at least two neighboring spins, which cannot be directly accessed in existing solid-state spectral measurements. Fortunately, this task was recently achieved by a distinct approach-the ultracold-atom based quantum simulator [73]. With the help of quantum gas microscopes, the direct measurement of instantaneous correlations, especially those high-order correlations, becomes possible [74-84]. The full spatial resolution of individual lattice sites has also been achieved. Furthermore, quantum simulators have control over dimensionality [85] and can engineer tailored optical potentials to precisely control model Hamiltonians [86,87]. As an example, an optical tweezer can be used to engineer a localized potential for holes, which allows for continuous control of a local impurity in a clean system [88]. This paradigmatic scenario of a single disordered lattice site with a tunable onsite potential will be analyzed in detail throughout this work.

Here, to systematically understand the high-order spin-hole correlations in the doped Hubbard model and the impact of an impurity on the correlations, we present an exact diagonalization calculation of $t-J-3 s$ and Hubbard models. Compared to the well-known spin polaron dressing in the case of a mobile dopant in a Mott insulator, we find that the presence of a local pinning field of variable strength results in significantly different spin correlation patterns. Such a difference is reflected in both third-order and fifth-order spin-hole correlation functions, suggesting a crossover from a spin-polaron surrounded by weakened magnetic correlations to a geometric defect surrounded by enhanced magnetic fluctuations. This work also complements our analysis of the fifth-order correlations of unpinned holes in Ref. [89]. Since the proposed correlation functions are composed of local spin and charge operators, they can be measured directly using the state-of-the-art quantum gas microscopy $[88,90]$, which can ultimately extend the quantitative conclusion to the thermodynamic limit.
This paper is organized as follows. We first introduce the models and methods used in Sec. II. Then we investigate the third-order and the fifth-order correlation functions in Secs. III and IV, respectively. We conclude our study in Sec. V. We discuss the differential third-order correlator in Appendix A and the finite-size effect in Appendix B.

\section{MODELS AND METHODS}

As the simplest microscopic model depicting Mott physics in a strongly correlated electronic system, the 2D (Fermi) Hubbard model is described by the Hamiltonian

$$
\mathcal{H}_{H}=-\sum_{\mathbf{i}, \mathbf{j}, \sigma}\left(t_{\mathbf{i} \mathbf{j}} \hat{c}_{\mathbf{j} \sigma}^{\dagger} \hat{\mathbf{c}}_{\mathbf{i} \sigma}+\text { H.c. }\right)+U \sum_{\mathbf{i}}\left(\hat{n}_{\mathbf{i} \uparrow}-\frac{1}{2}\right)\left(\hat{n}_{\mathbf{i} \downarrow}-\frac{1}{2}\right),
$$

where $\hat{c}_{\mathbf{i} \sigma}^{\dagger}\left(\hat{c}_{\mathbf{i} \sigma}\right)$ and $\hat{n}_{\mathbf{i} \sigma}$ denote the creation (annihilation) and density operator, respectively, at site $\mathbf{i}$ of $\operatorname{spin} \sigma ; t_{\mathbf{i j}}$ is the hopping, restricted here to nearest neighbors (nn) $t_{\langle\mathbf{i j}\rangle}=t$. As mentioned above, the single-band Hubbard model is believed to capture the essential physics of strongly correlated materials such as cuprates $[2,3]$ and can be precisely simulated by the cold-atom experiments [81-83,91-95].

The Hubbard model's Hilbert space dimension is relatively large, limiting both the system size and the number of states accessible in exact numerical calculations. To investigate the temperature dependence in the regime accessible by coldatom experiments (typically $T \sim 0.5 t$ ), we also consider the low-energy approximation of the Hubbard model near halffilling-the $t$ - $J$-like spin model. Through a $t / U$ expansion to the lowest order, one can simplify the Hubbard model to the $t$ - $J$ model [96-99]:

$$
\mathcal{H}_{t-J}=-t \sum_{\langle\mathbf{i}, \mathbf{j}\rangle, \sigma}\left(\tilde{c}_{\mathbf{j} \sigma}^{\dagger} \tilde{c}_{\mathbf{i} \sigma}+\text { H.c. }\right)+J \sum_{\langle\mathbf{i}, \mathbf{j}\rangle}\left(\mathbf{S}_{\mathbf{i}} \cdot \mathbf{S}_{\mathbf{j}}-\frac{\hat{n}_{\mathbf{i}} \hat{n}_{\mathbf{j}}}{4}\right),
$$

where $\mathbf{S}_{\mathbf{i}} \cdot \mathbf{S}_{\mathbf{j}}=\hat{S}_{\mathbf{i}}^{z} \hat{S}_{\mathbf{j}}^{z}+\frac{1}{2}\left(\hat{S}_{\mathbf{i}}^{+} \hat{S}_{\mathbf{j}}^{-}+\hat{S}_{\mathbf{i}}^{-} \hat{S}_{\mathbf{j}}^{+}\right)$, with $\hat{S}_{\mathbf{i}}^{z}=\left(\hat{n}_{\mathbf{i} \uparrow}-\hat{n}_{\mathbf{i} \downarrow}\right) /$ 2 and $\hat{S}_{\mathbf{i}}^{+}=\left(\hat{S}_{\mathbf{i}}^{-}\right)^{\dagger}=\tilde{c}_{\mathbf{i} \uparrow}^{\dagger} \tilde{c}_{\mathbf{i} \downarrow}$. The constrained fermionic operators acting in the Hilbert space without double occupancy are defined as $\tilde{c}_{\mathbf{i} \sigma}^{\dagger}=\hat{c}_{\mathbf{i} \sigma}^{\dagger}\left(1-\hat{n}_{\mathbf{i} \bar{\sigma}}\right)$. Although sometimes negligible, there is a three-site term at the same lowest order $\left(\sim t^{2} / U\right)$ that contributes to the dopant's motion $[38,39,98-$ 108]. This defines the $t-J-3 s$ model with the Hamiltonian given by $\mathcal{H}_{t-J-3 s}=\mathcal{H}_{t-J}+\mathcal{H}_{3 s}$ :

$$
\mathcal{H}_{3 s}=-\frac{J}{4} \sum_{\substack{\left.\left\langle\mathbf{i} \mathbf{j}, \mathbf{j}, \mathbf{i} \mathbf{i}^{\prime}\right\}^{\prime}\right\rangle \\ \mathbf{j} j \mathbf{j}^{\prime}, \sigma}}\left(\tilde{c}_{\mathbf{j}^{\prime} \sigma}^{\dagger} \tilde{n}_{\mathbf{i} \bar{\sigma}} \tilde{c}_{\mathbf{j} \sigma}+\tilde{c}_{\mathbf{j}^{\prime} \sigma}^{\dagger} \tilde{c}_{\mathbf{i} \bar{\sigma}}^{\dagger} \tilde{c}_{\mathbf{i} \sigma} \tilde{c}_{\mathbf{j} \bar{\sigma}}\right) .
$$

We use both the Hubbard and $t-J-3 s$ models to study highorder correlations, where the latter allows for access to higher temperatures. Comparisons between the two models allow identifying which effects are captured by effective spin models and do not require higher-order expansions in $t / U$. Unless otherwise specified, we use $U=8 t$ for the Hubbard model and accordingly $J=0.5 t$ for the $t-J-3 s$ model throughout this paper.

To test the correlations with respect to an immobile hole, we also consider an extra pinning potential in the origin $\left(\mathbf{r}_{0}=\mathbf{0}\right)$, controlling the mobility of the single hole. The 
Hamiltonian becomes

$$
\mathcal{H}=\mathcal{H}_{0}+V \sum_{\sigma} \hat{n}_{\mathbf{0} \sigma},
$$

where $\mathcal{H}_{0}$ is the Hubbard or $t-J-3 s$ Hamiltonian, and $V$ is the strength of a local pinning potential. This pinning potential can be realized experimentally by an optical tweezer in an ultracold atom system [88]. Hence, the high-order correlations analyzed in this paper and Ref. [89] are directly accessible to these experiments. Note that the strength of spin-exchange and three-site terms in the $t-J$ model involving a virtually doubly-occupied central site (which is perturbed by the added pinning potential) must be modified when $|V|$ becomes comparable to the Hubbard interaction $U$. Throughout this work, we neglect such corrections and focus on the regime $|V| \leqslant U$. The difference between Hubbard and $t-J-3 s$ models are quantitatively compared and discussed whenever relevant.

To resolve the wave function and the corresponding correlators over a range of low temperatures in a $2 \mathrm{D} D_{4}$ symmetric system, we perform exact diagonalization calculations on a $4 \times 4$ cluster with periodic boundary conditions. Throughout this paper, we evaluate the expectation values of observables in a canonical ensemble. For convenience, we denote the expectation as the thermal average

$$
\langle\hat{O}\rangle=\operatorname{Tr}\left[\frac{e^{-\mathcal{H} / T}}{\mathcal{Z}} \hat{O}\right] \approx \sum_{n<n_{\max }} \frac{e^{-E_{n} / T}}{\mathcal{Z}}\langle n|\hat{O}| n\rangle,
$$

in which $\mathcal{Z}$ is the partition function. The $n_{\max }$ sets the numerical truncation of excited states, which satisfies $E_{n_{\max }}-E_{0} \gg$ $T$ for all temperatures considered in our work. In the results presented in Secs. III and IV, we keep $n_{\max } \sim 650$ states for the Hubbard model, giving reliable results up to $T \sim 0.4 t$, whereas $n_{\max } \sim 13,000$ states for the $t-J-3 s$ model, giving reliable results up to $T \sim t$.

In this paper, we employ the parallel Arnoldi and $\mathrm{Pa}-$ radeisos algorithm to determine the equilibrium ground-state wave function and expectation values [109,110]. Unless otherwise indicated, we include all total $-S^{z}$ sectors in the thermal ensemble. Part of the results in Sec. IV are benchmarked using the density matrix renormalization group (DMRG) at zero temperature in a $6 \times 12$ cluster with cylindrical boundary condition, to investigate possible artifacts caused by the finite system size. We used bond dimension $\chi=2000$ for these DMRG simulations, which leads to the truncation error $3.83 \times 10^{-6}$ for $V=0$ and $2.38 \times 10^{-7}$ for $V=5 t$. We also use the determinant quantum Monte Carlo (DQMC) $[111,112]$ to benchmark the finite-size effects in the Appendix, using $6 \times 6$ and $8 \times 8$ clusters at high temperatures.

\section{THE THIRD-ORDER SPIN-HOLE CORRELATIONS}

To describe the spin correlations with respect to a doped hole, we consider the following third-order correlation function:

$$
B\left(\mathbf{r}, \mathbf{r}^{\prime}, \mathbf{d}\right)=4\left\langle\hat{n}_{\mathbf{r}}^{h} \hat{S}_{\mathbf{r}^{\prime}}^{z} \hat{S}_{\mathbf{r}^{\prime}+\mathbf{d}}^{z}\right\rangle /\left\langle\hat{n}_{\mathbf{r}}^{h}\right\rangle,
$$

where the hole density operator $\hat{n}_{\mathbf{r}}^{h}=\left(1-\hat{n}_{\mathbf{r} \uparrow}\right)\left(1-\hat{n}_{\mathbf{r} \downarrow}\right)$. In this paper, we focus on the $|\mathbf{d}|=1$ (neighboring spins) and $|\mathbf{d}|=\sqrt{2}$ (diagonal spins), allowing the relative distance be-

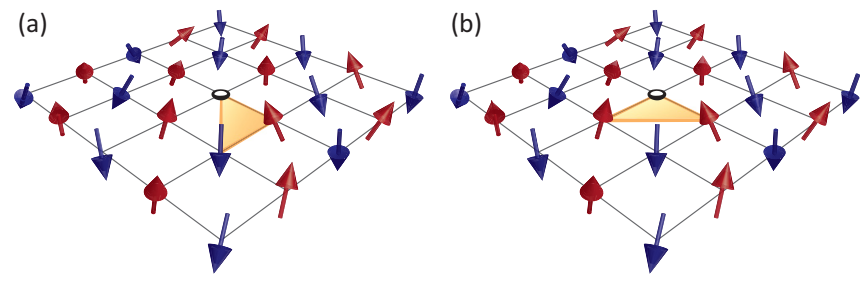

FIG. 1. Schematic showing the third-order spin-hole correlators for (a) nearest spins $(|\mathbf{d}|=1)$ and (b) next-nearest spins $(|\mathbf{d}|=\sqrt{2})$.

tween the hole and the spin "bond" $\left|\mathbf{r}-\mathbf{r}^{\prime}\right|$ to vary (see Fig. 1).

Figure 2 gives an overview of the spatial $\left(\mathbf{r}^{\prime}-\mathbf{r}\right)$ distribution of the $B\left(\mathbf{r}, \mathbf{r}^{\prime}, \mathbf{d}\right)$ in a single-hole-doped Hubbard model at zero temperature, with $\mathbf{r}$ corresponding to the coordinate where the pinning potential $V$ is applied (white dot). For different pinning potentials, the nearest-neighbor $(|\mathbf{d}|=1)$ correlations are always negative, while the diagonal $(|\mathbf{d}|=$ $\sqrt{2}$ ) correlations are mostly positive due to the strong AFM order. However, the spatial variation of these correlators indicates different underlying physics triggered by the pinning potential, as discussed below.

We first consider systems with a mobile hole, i.e., for $V=$ 0 . As shown in the left panel of Fig. 2(a), the (absolute value of the) third-order correlator $B\left(\mathbf{r}, \mathbf{r}^{\prime}, \mathbf{d}\right)$ is weakened for shorter distances $\left|\mathbf{r}-\mathbf{r}^{\prime}\right|$. This spatial distribution can be understood in terms of the spin polaron [113,114], where a dopant's motion is dressed by a cloud of spin defects, forming a polaronic quasiparticle. As the system is translationally invariant for
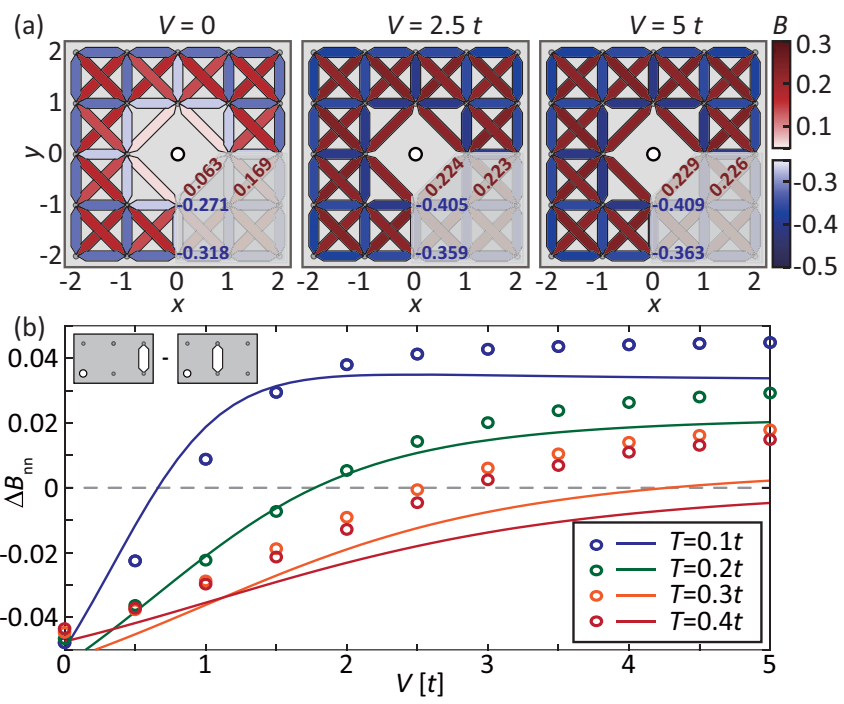

FIG. 2. (a) The spatial distribution of $B\left(\mathbf{r}, \mathbf{r}^{\prime}, \mathbf{d}\right)$ for $|\mathbf{d}|=1$ and $|\mathbf{d}|=\sqrt{2}$ in a $n=1 / 16$ doped Hubbard model at $T=0$, with pinning potential $V=0, V=2.5 t$, and $V=5 t$, respectively. The numbers in the bottom-right corner of each panel mark the values of the correlators mentioned in the main text. (b) The $V$-dependence of the differential third-order correlators $\Delta B_{\mathrm{nn}}$ for $T=0.1 t$ (blue), $0.2 t$ (green), $0.3 t$ (orange), and $0.4 t$ (red). The dots and solid lines denote the results obtained for the Hubbard and $t-J-3 s$ model, respectively. The inset in panel (b) explains the definition of the differential correlators. 
$V=0$, the correlator $B\left(\mathbf{r}, \mathbf{r}^{\prime}, \mathbf{d}\right)$ depicts the concentration of spin defects in the comoving frame of the mobile hole. Due to the small radius of the spin polaron (local screening of spin correlations), the amplitudes of correlators for large $\left|\mathbf{r}^{\prime}-\mathbf{r}\right|$ 's asymptotically approach those of an undoped AFM system.

In contrast to the free hole, the spin correlations in the presence of a pinning potential $V>0$ lead to significantly different patterns. With the pinning potential applied at site $\mathbf{r}$, we examine the the third-order correlator $B\left(\mathbf{r}, \mathbf{r}^{\prime}, \mathbf{d}\right)$ between the hole (at $\mathbf{r}$ ) and the spins at $\mathbf{r}^{\prime}$ and $\mathbf{r}^{\prime}+\mathbf{d}$. As shown in the right two panels of Figs. 2(a) and 2(b), $B\left(\mathbf{r}, \mathbf{r}^{\prime}, \mathbf{d}\right)$ becomes stronger for shorter distances $\left|\mathbf{r}-\mathbf{r}^{\prime}\right|$ (i.e., for $|\mathbf{d}|=1$ it becomes more negative, while for $|\mathbf{d}|=\sqrt{2}$ it becomes more positive). To distinguish from the screening of correlations in the spin-polaron picture, we denote this phenomenon as "antiscreening." A similar phenomena has recently been observed in experiments, albeit at higher temperatures, where it has been attributed to imperfections of the pinning potential [88]. Indirectly, this phenomenon is also consistent with the increased local moments measured in NMR and $\mu \mathrm{SR}$ experiments in Zn-substituted cuprates [56-59]. One can intuitively understand this phenomenon by observing the fact that spin correlations in systems with a lower coordination numbers (e.g., a 1D chain) are stronger than in the $2 \mathrm{D}$ plane with otherwise identical parameters due to the existence of reduced frustrations (an AFM state favors spin to form singlets with all its nearest neighbors) $[115,116]$. Thus, a system with a strong pinning potential tends to form a $1 \mathrm{D}$ edge at the boundary surrounding the impurity. Here the system can lower its energy by forming stronger singlet bonds closer to the impurity, while retaining the bulk spin order at further-away sites. In addition to the change in spatial distribution, the presence of a pinning potential also leads to an overall enhancement of all correlators (in terms of absolute values).

To quantify the different distributions of the third-order correlators, we define the difference between the nearestneighbor correlators as [see the illustration in the inset of Fig. 2(b)]

$$
\Delta B_{\mathrm{nn}}=B(\mathbf{r}, \mathbf{r}+2 \hat{x}, \hat{y})-B(\mathbf{r}, \mathbf{r}+\hat{x}, \hat{y}) .
$$

Through the difference, the uniform (positive or negative) background in the "bare" correlator $B\left(\mathbf{r}, \mathbf{r}^{\prime}, \mathbf{d}\right)$ is removed. Thus, we refer to the $\Delta B_{\mathrm{nn}}$ as differential third-order correlators. As shown in Fig. 2(b), $\Delta B_{\mathrm{nn}}$ is always negative for $V=0$, indicating the screening effect of the spin polaron; the $\Delta B_{\text {nn }}$ turns to positive for a finite $V$, indicating the onset of antiscreening [117]. As such, the crossover between screening and antiscreening natures is clearly reflected by the differential third-order correlators, while the original $B\left(\mathbf{r}, \mathbf{r}^{\prime}, \hat{y}\right) \mathrm{s}$ do not have a sign change. The reason is that the original ones contain a substantial contribution from the lower-order correlators (i.e., $\left.\left\langle\hat{S}_{\mathbf{r}}^{z} \hat{S}_{\mathbf{r}+\hat{y}}^{z}\right\rangle\right)$, which is sizably negative. A spatial difference with respect to the hole, however, cancels (or at least heavily reduces) this lower-order background.

The above observation indicates that a "genuine" correlator is required while describing the underlying many-body physics. Such a genuine third-order correlator can be more intuitively defined as the connected part of $B\left(\mathbf{r}, \mathbf{r}^{\prime}, \mathbf{d}\right)$

$$
B^{c}\left(\mathbf{r}, \mathbf{r}^{\prime}, \mathbf{d}\right)=B\left(\mathbf{r}, \mathbf{r}^{\prime}, \mathbf{d}\right)-4 \sum_{\mathbf{r}^{\prime \prime}}\left\langle\hat{S}_{\mathbf{r}^{\prime \prime}}^{z} \hat{S}_{\mathbf{r}^{\prime \prime}+\mathbf{d}}^{z}\right\rangle / N,
$$

where $N$ denotes the number of lattice sites. As such, the $B^{c}\left(\mathbf{r}, \mathbf{r}^{\prime}, \mathbf{d}\right)$ reflects the net hole-spin-spin correlation on top of the AFM background, without the need to extract through a spatial difference. As shown in Fig. 3(a), the $B^{c}\left(\mathbf{r}, \mathbf{r}^{\prime}, \mathbf{d}\right)$ 's for small $\left|\mathbf{r}-\mathbf{r}^{\prime}\right|$ flip sign in the presence of the pinning potential, which allows for a better discrimination of the screening and antiscreening regimes.

Given that thermal fluctuations are expected to disrupt long-range ordering, the crossover between the screening and antiscreening situations should depend on both temperature and the pinning potential. To address this, we calculate the $T$ - and $V$-dependence of $B^{c}\left(\mathbf{r}, \mathbf{r}^{\prime}, \mathbf{d}\right)$. As mentioned in Sec. II, the Hilbert space size of the Hubbard model limits the accessible maximal temperature; therefore, we also calculate the above correlators for the $t-J-3 s$ model. As shown in Figs. 3(b) and 3(c), the $B^{c}$ characterizes a crossover from a screening (negative) to the antiscreening (positive) regime for any temperature $T<0.3 t$. The critical $V$ value increases with temperature, because thermal fluctuations help with the hole's delocalization and, therefore, its mobility becomes larger for the same strength of pinning potential.

By comparing the Hubbard and $t-J-3 s$ models in Fig. 3(c), we note that the results obtained from both models agree quantitatively at the small $V$ side, suggesting that the thirdorder spin-hole correlations originate from hole motion in an AFM background instead of any interactions beyond the $\mathcal{O}\left(t^{2} / U\right)$. On the large $V$ side, $B^{c}\left(\mathbf{r}, \mathbf{r}^{\prime}, \mathbf{d}\right)$ displays slightly different temperature dependence for two models, which can be attributed to the modified exchange coupling in the vicinity of the pinning site: Between this site and its nearest neighbors, the superexchange coupling becomes $J_{\text {eff }}=$ $J U^{2} /\left(U^{2}-V^{2}\right)>J$. At much higher temperatures $(T>J)$, thermal fluctuations smear out the AFM spin correlations and thus suppress the third-order correlator.

\section{THE FIFTH-ORDER RING-SPIN CORRELATORS}

The third-order correlators $B\left(\mathbf{r}, \mathbf{r}^{\prime}, \mathbf{d}\right)$ reflect the spin fluctuations around the hole. These correlators are expected to be weak for mobile holes because the underlying motion of the dopants admixes spins from different sublattices in the surrounding AFM [113,118]. To minimize the effect of averaging over different trajectories and provide deeper insights into the underlying spin-charge correlation, one may consider constructing higher-order correlators. To this end, we examine the (fifth-order) ring-spin correlators with respect to a doped hole, which is introduced recently in Ref. [89] as

$$
C_{\diamond}(\mathbf{r}, \mathbf{d})=2^{4}\left\langle\hat{n}_{\mathbf{r}}^{h} \hat{S}_{\mathbf{r}+\mathbf{d}+\hat{x}}^{z} \hat{S}_{\mathbf{r}+\mathbf{d}+\hat{y}}^{z} \hat{S}_{\mathbf{r}+\mathbf{d}-\hat{x}}^{z} \hat{S}_{\mathbf{r}+\mathbf{d}-\hat{y}}^{z}\right\rangle /\left\langle\hat{n}_{\mathbf{r}}^{h}\right\rangle .
$$

As illustrated in Fig. 4, this correlator reflects the ring-spin correlations in a comoving frame of the doped hole. By including nonzero displacements $\mathbf{d}$ (between the ring-center and the hole), Eq. (9) generalizes the five-point correlators introduced in Ref. [89].

We first investigate the dependence of $C_{\diamond}(\mathbf{r}, \mathbf{d})$ on the ring displacement $\mathbf{d}$, and consider a mobile hole with no pinning potential $(V=0)$. In this case, the system has translational invariance and $C_{\diamond}(\mathbf{r}, \mathbf{d})$ is only a function of $\mathbf{d}$. As shown in Fig. 5(a) and the top-left panel of Fig. 5(b), $C_{\diamond}$ is positive for any $|\mathbf{d}|>1$, but becomes sizable and negative for $\mathbf{d}=\mathbf{0}$. 

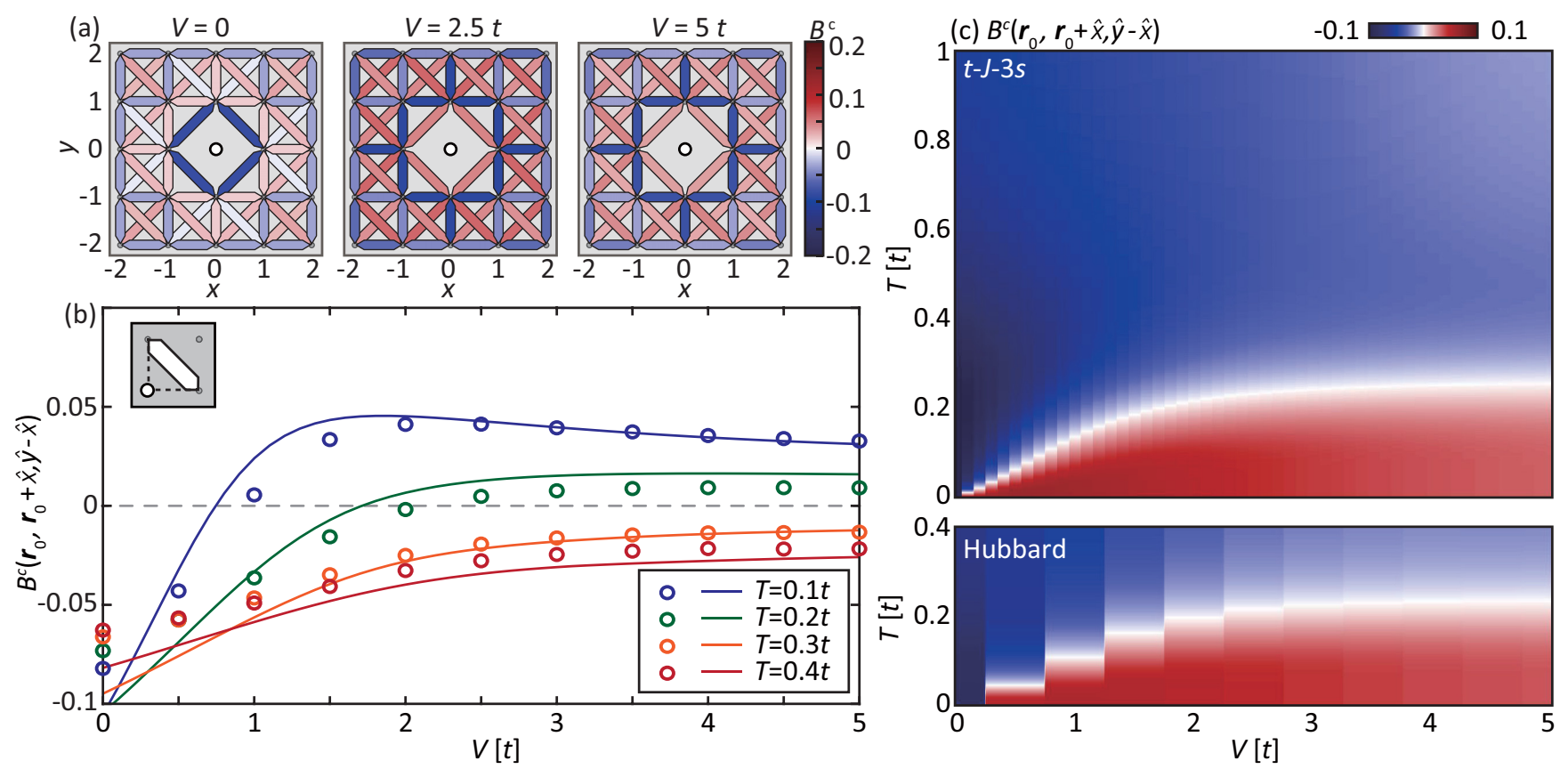

FIG. 3. (a) The spatial distribution of connected parts $B^{c}\left(\mathbf{r}, \mathbf{r}^{\prime}, \mathbf{d}\right)$ in a $n=1 / 16$ doped Hubbard model at $T=0$, with pinning potential $V=0, V=2.5 t$ and $V=5 t$, respectively. (b) The $V$-dependence of the connected third-order correlators $B^{c}\left(\mathbf{r}, \mathbf{r}^{\prime}, \mathbf{d}\right)$ for $T=0.1 t$ (blue), $0.2 t$ (green), $0.3 t$ (orange), and $0.4 t$ (red). The dots and solid lines denote the results obtained for the Hubbard and $t-J-3 s$ model, respectively. The inset in panel (b) explains the definition of the differential correlators. (c) The temperature and pinning potential dependence of the genuine third-order correlator $B^{c}\left(\mathbf{r}_{0}, \mathbf{r}_{0}+\hat{x}, \mathbf{d}\right)$ with $|\mathbf{d}|=\hat{y}-\hat{x}$.

This behavior is reminiscent of a $\mathbb{Z}_{2}$ Gauss law expected in a string description of the single spin polaron: when the mobile hole forms a spin polaron, its motion creates a "string" of displaced spins; In the $C_{\diamond}$ correlator, spins which are part of the string originate from the opposite sublattice and contribute a negative sign [89]. Since $C_{\diamond}(\mathbf{r}, \mathbf{0})$ involves only one site belonging to the string, it is expected to be negative; whereas $C_{\diamond}(\mathbf{r}, \mathbf{d} \neq \mathbf{0})$ has statistically more contributions from an even number of spins affected by a string, displaying a positive value. We find they reach a quantitative agreement by comparing the $t-J$ model and the Hubbard model with different system sizes. This indicates that the observed correlations can be understood from the spin-exchange picture, while higher-order terms in the $t / U$ expansion can be ignored. The consistency between the DMRG calculation in a sizable system and the ED calculation in a $4 \times 4$ cluster further precludes the influence of system size and models.

We then consider systems with a finite pinning potential $V$ in the center at site $\mathbf{r}_{0}$. As shown in the upper panel of

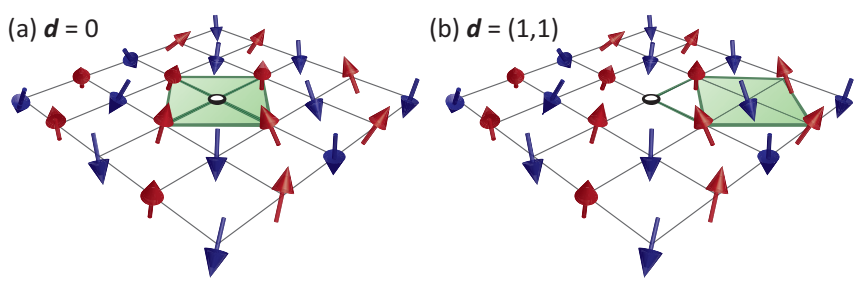

FIG. 4. Schematic showing the fifth-order ring-spin correlators for (a) $\mathbf{d}=(0,0)$ and (b) $\mathbf{d}=(1,1)$.
Fig. 5(b), the onsite $(\mathbf{d}=\mathbf{0})$ correlator $C_{\diamond}\left(\mathbf{r}_{0}, \mathbf{0}\right)$ flips sign with a $V$-field. This is a direct consequence of a hole becoming immobile: when the pinning potential traps the dopant hole, the spin polaron breaks down, and the remaining system becomes a half-filled AFM system with a static defect. Since next-nearest spins are expected to be parallel in a pure Néel state, the $C_{\diamond}(\mathbf{r}, \mathbf{0})$ becomes positive in this immobile case. This difference induced by the hole's mobility further reflects the screening to antiscreening crossover, consistent with the observation using the third-order correlators in Sec. III.

The sensitivity to a pinning potential is affected by the thermal fluctuation at finite temperature. As shown in the lower panel of Fig. 5(b), the onsite correlator $C_{\diamond}\left(\mathbf{r}_{0}, \mathbf{0}\right)$ remains negative until $V \sim 2.5 t$ at a higher temperature $T=0.4 t$. As mentioned in Sec. III, this is a consequence of the hole's delocalization enhanced by thermal fluctuations. To better visualize this crossover, we extract the $C_{\diamond}\left(\mathbf{r}_{0}, \mathbf{0}\right)$ and plot its $T$ and $V$ dependence in Figs. 6(a) and 6(b). As temperatures rise, the regime dominated by the spin-polaron screening (blue) extends to a larger pinning potential. We observe a similar crossover here, as in Fig. 3; note that the crossover's exact position may be different for different observables as it is not a phase transition. Except for this crossover, we find that all $C_{\diamond}\left(\mathbf{r}_{0}, \mathbf{d}\right)$ s decrease with increasing temperature, which occurs dramatically near $T \sim J$ and indicates the thermal melting of the surrounding antiferromagnet. Given the presence of four spins in the fifth-order correlator, its temperature dependence is more evident than the third-order one presented in Fig. 3(c).

As such, the temperature dependence of this fifth-order correlator reflects the screening to antiscreening crossover 
(a) $t-J$ model by DMRG $(T=0)$
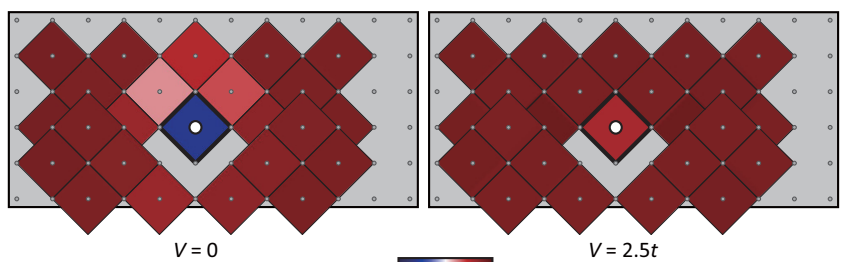

(b) Hubbard model by ED
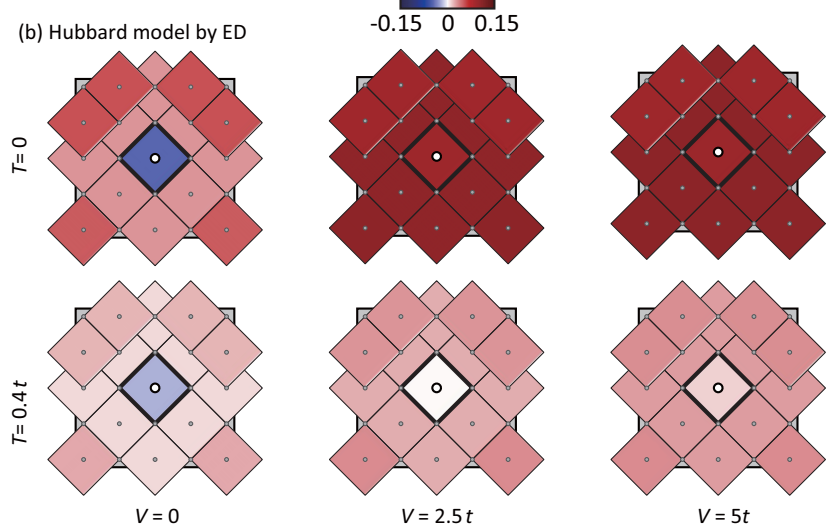

FIG. 5. (a) The distribution of $C_{\diamond}(\mathbf{r}, \mathbf{d})$ for different distances $\mathbf{d}$, $\mathbf{r}$ in the center with $V=0$ (left) and $V=2.5 t$ (right), calculated for a $6 \times 12 t-J$ model at zero temperature by DMRG. The weak asymmetry in the $x$-direction is caused by the noncentral location of the hole at $x=6$, while the system is translational invariant along the $y$ direction due to the cylindrical boundary condition. (b) The distribution of $C_{\diamond}\left(\mathbf{r}=\mathbf{r}_{0}, \mathbf{d}\right)$ for different distances $\mathbf{d}$, strengths $V$ of the pinning potential in the center at $\mathbf{r}_{0}$, and temperatures $T$, calculated for the Hubbard model in a $4 \times 4$ cluster. The central white circle denotes the position of the dopant hole $\mathbf{r}_{0}$ on the site of the pinning potential.

similar to the third-order one. The difference is that this phenomenon has already been reflected in the "bare" fifth-order correlator, without spatial derivative or subtracting disconnected parts. In contrast, the genuine (connected) fifth-order correlator reflects a different level of information. To extract the genuine fifth-order correlators, we subtract off the disconnected pieces of the correlator following the same manner as Ref. [89]:

$$
\begin{aligned}
C_{\diamond}^{c}(\mathbf{r}, \mathbf{d})= & C_{\diamond}(\mathbf{r}, \mathbf{d}) \\
& -2^{4}\left[\sum_{\mathbf{l} \notin(\mathbf{i}, \mathbf{j}, \mathbf{k})} \frac{\left\langle\hat{n}_{\mathbf{r}}^{\mathrm{h}} \hat{S}_{\mathbf{r}+\mathbf{d}+\mathbf{i}}^{z} \hat{S}_{\mathbf{r}+\mathbf{d}+\mathbf{j}}^{z} \hat{S}_{\mathbf{r}+\mathbf{d}+\mathbf{k}}^{z}\right\rangle}{\left\langle\hat{n}_{\mathbf{r}}^{\mathrm{h}}\right\rangle} \frac{\left\langle\hat{n}_{\mathbf{r}}^{\mathrm{h}} \hat{S}_{\mathbf{r}+\mathbf{d}+\mathbf{l}}^{z}\right\rangle}{\left\langle\hat{n}_{\mathbf{r}}^{\mathrm{h}}\right\rangle}\right. \\
& +\sum_{(\mathbf{i}, \mathbf{j}) \notin(\mathbf{k}, \mathbf{l})} \frac{\left\langle\hat{n}_{\mathbf{r}}^{\mathrm{h}} \hat{S}_{\mathbf{r}+\mathbf{d}+\mathbf{i}}^{z} \hat{S}_{\mathbf{r}+\mathbf{d}+\mathbf{j}}^{z}\right\rangle}{\left\langle\hat{n}_{\mathbf{r}}^{\mathrm{h}}\right\rangle} \frac{\left\langle\hat{n}_{\mathbf{r}}^{\mathrm{h}} \hat{S}_{\mathbf{r}+\mathbf{d}+\mathbf{k}}^{z} \hat{S}_{\mathbf{r}+\mathbf{d}+\mathbf{l}}^{z}\right\rangle}{\left\langle\hat{n}_{\mathbf{r}}^{\mathrm{h}}\right\rangle} \\
& +\sum_{\mathbf{i} \neq \mathbf{j} \notin(\mathbf{k}, \mathbf{l})} \frac{\left\langle\hat{n}_{\mathbf{r}}^{\mathrm{h}} \hat{S}_{\mathbf{r}+\mathbf{d}+\mathbf{i}}^{z}\right\rangle}{\left\langle\hat{n}_{\mathbf{r}}^{\mathrm{h}}\right\rangle} \frac{\left\langle\hat{n}_{\mathbf{r}}^{\mathrm{h}} \hat{S}_{\mathbf{r}+\mathbf{d}+\mathbf{j}}^{z}\right\rangle}{\left\langle\hat{n}_{\mathbf{r}}^{\mathrm{h}}\right\rangle} \frac{\left\langle\hat{n}_{\mathbf{r}}^{\mathrm{h}} \hat{S}_{\mathbf{r}+\mathbf{d}+\mathbf{k}}^{z} \hat{S}_{\mathbf{r}+\mathbf{d}+\mathbf{l}}^{z}\right\rangle}{\left\langle\hat{n}_{\mathbf{r}}^{\mathrm{h}}\right\rangle} \\
& \left.+\frac{\left\langle\hat{n}_{\mathbf{r}}^{\mathrm{h}} \hat{S}_{\mathbf{r}+\mathbf{d}+\hat{x}}^{z}\right\rangle}{\left\langle\hat{n}_{\mathbf{r}}^{\mathrm{h}}\right\rangle} \frac{\left\langle\hat{n}_{\mathbf{r}}^{\mathrm{h}} \hat{S}_{\mathbf{r}+\mathbf{d}+\hat{y}}^{z}\right\rangle}{\left\langle\hat{n}_{\mathbf{r}}^{\mathrm{h}}\right\rangle} \frac{\left\langle\hat{n}_{\mathbf{r}}^{\mathrm{h}} \hat{S}_{\mathbf{r}+\mathbf{d}-\hat{x}}^{z}\right\rangle}{\left\langle\hat{n}_{\mathbf{r}}^{\mathrm{h}}\right\rangle} \frac{\left\langle\hat{n}_{\mathbf{r}}^{\mathrm{h}} \hat{S}_{\mathbf{r}+\mathbf{d}-\hat{y}}^{z}\right\rangle}{\left\langle\hat{n}_{\mathbf{r}}^{\mathrm{h}}\right\rangle}\right] .
\end{aligned}
$$

Figures 6(c) and 6(d) show the dependence of the connected correlator $C_{\diamond}^{c}(\mathbf{r}, \mathbf{0})$ on the temperature and pinning potential. We find that this correlator is sizably negative even for large pinning potentials. Although the characteristic temperature, above which $\left|C_{\diamond}^{c}\right|$ drops dramatically, decreases slightly for the systems with pinned holes, the absolute value of $C_{\diamond}^{c}$ does not change much with $V$. This observation indicates that the genuine fifth-order correlator is always present, significantly deviating from the classical Néel state regardless of whether the hole is mobile. Such an intrinsic nature is invisible in lower-order correlators.

In the remainder of this section, we elucidate the physical origin and properties of the genuine fifth-order ring-spin correlators. We discuss the role of different statistical ensembles on the fifth-order ring-spin correlators.

To this end, we compare a spin-imbalanced ensemble with definite total $\operatorname{spin} S^{z}=1 / 2$ to a spin-balanced statistical mixture with $\left\langle\hat{S}^{z}\right\rangle=0$. Alternatively, in the former case, we could consider an ensemble with definite $S^{z}=-1 / 2$, which gives
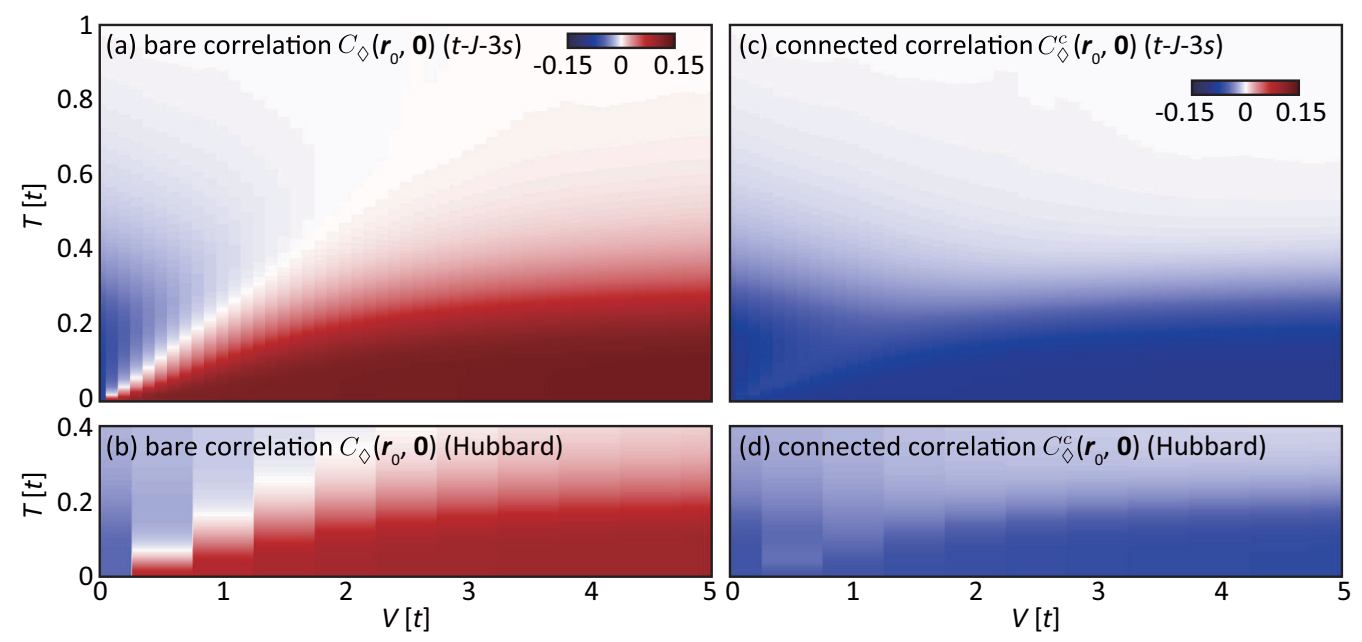

FIG. 6. (a), (b) The bare onsite ring-spin correlator $C_{\diamond}\left(\mathbf{r}_{0}, \mathbf{d}=\mathbf{0}\right)$ as a function of pinning potential $V$ and temperature $T$, calculated using the (a) $t-J-3 s$ model and (b) Hubbard model. (c), (d) Same as (a), (b) but for the connected part of the onsite ring-spin correlator $C_{\diamond}^{c}\left(\mathbf{r}_{0}, \mathbf{d}=\mathbf{0}\right)$ as a function of pinning potential $V$ and temperature $T$. 
the same results: the higher-order correlators $C_{\diamond}$ and $C_{\diamond}^{c}$ each involve an even number of spin operators $\hat{S}^{z}$. Moreover, the underlying spin or Hubbard Hamiltonian is invariant under a global spin-flip $\left(S_{j}^{z} \rightarrow-S_{j}^{z}\right)$. Hence, the correlators in the sectors with definite $S^{z}= \pm 1 / 2$ are equivalent,

$$
\left.C_{\diamond}\right|_{S^{z}=1 / 2}=\left.C_{\diamond}\right|_{S^{z}=-1 / 2},\left.\quad C_{\diamond}^{c}\right|_{S^{z}=1 / 2}=\left.C_{\diamond}^{c}\right|_{S^{z}=-1 / 2} .
$$

With the same argument, in the spin-balanced ensemble, $\left\langle\hat{S}^{z}\right\rangle=0$, all lower-order correlators involving an odd number of spin operators vanish. While an ensemble with definite $S^{z}=1 / 2$ or $-1 / 2$ is hard to realize in a solid-state system, both spin ensembles can be addressed experimentally with ultracold atoms and with full spin and charge resolution $[119,120]$. This ensemble can be achieved by postselecting experimental data with specific $S^{z}$ values.

The two spin ensembles correspond to different ways of taking the zero-temperature limit. The usual canonical ensemble converges to a balanced mixture of the two sectors $S^{z}=+1 / 2$ and $S^{z}=-1 / 2$ when $T \rightarrow 0$. In the resulting spin-balanced ensemble $\left\langle\hat{S}^{z}\right\rangle=0$, and all but the second sum on the right-hand side of Eq. (10) vanish. In contrast, for the ground state with a definite $S^{z}=1 / 2$, all terms in Eq. (10) contribute.

Nevertheless, for a mobile hole we find that the calculated connected correlators do not change significantly if switched to the imbalanced ensemble: In Fig. 7(a), we plot $\left.C_{\triangle}^{c}\right|_{\left\langle S^{z}\right\rangle=0}$ as a function of $t / J$ (see Fig. 1 in Ref. [89]) and compare it to the spin imbalanced ensemble. Qualitatively, the same behavior is found for all considered values of $t / J$. The deviations are largest for small values of $t / J$, where we expect a smaller spin-polaron radius in the ground state [113].

The situation becomes slightly different when the hole is trapped by a pinning potential. In Fig. 7 we compare connected and bare correlators for a spin-imbalanced ensemble with the fixed total $S^{z}=1 / 2$. The bare correlator $C_{\diamond}$ in Fig. 7(b) reflects a crossover similar to Fig. 6(a), with slightly stronger temperature dependence. This sensitivity to temperature results from the fact that the fixed total spin $S^{z}=1 / 2$ gives finite contributions to the odd terms in Eq. (10) as lower orders and thus induces more fluctuations when temperature increases. For the same reason, the connected fifth-order correlator $C_{\diamond}^{c}$ also displays a slightly stronger sensitivity to the pinning potential $V \mathrm{~s}$ [see Fig. 7(b)].

Most significantly, we observe in Fig. 7(c) that the connected correlator $C_{\diamond}^{c}$ decreases in magnitude in the spinimbalanced ensemble for large $V$ and small temperatures $T$; in contrast, it remains sizably negative in this regime for the spinbalanced ensemble [see Fig. 6(c)]. We attribute this influence of the spin ensemble on the connected higher-order correlator to the formation of Néel order and a nonzero staggered magnetization, $\left\langle\hat{M}^{z}\right\rangle=\sum_{\mathbf{r}}(-1)^{r_{x}+r_{y}}\left\langle\hat{S}_{\mathbf{r}}^{z}\right\rangle \neq 0$, in the lattice. In a finite-size system, like the one we study, the latter requires spin-imbalance and a pinning potential for the hole to avoid mixing of A- and B- sublattices in the comoving frame of the hole where we define the higher-order correlators. When a nonzero staggered magnetization forms in the comoving frame with the hole, the lower-order terms-e.g., $\left\langle\hat{S}_{\mathbf{r}}^{z}\right\rangle$-provide sizable nonvanishing contributions to the bare ring-spin correlators. Since we observe that bare ring-spin correlators are only weakly affected by different spin ensem-
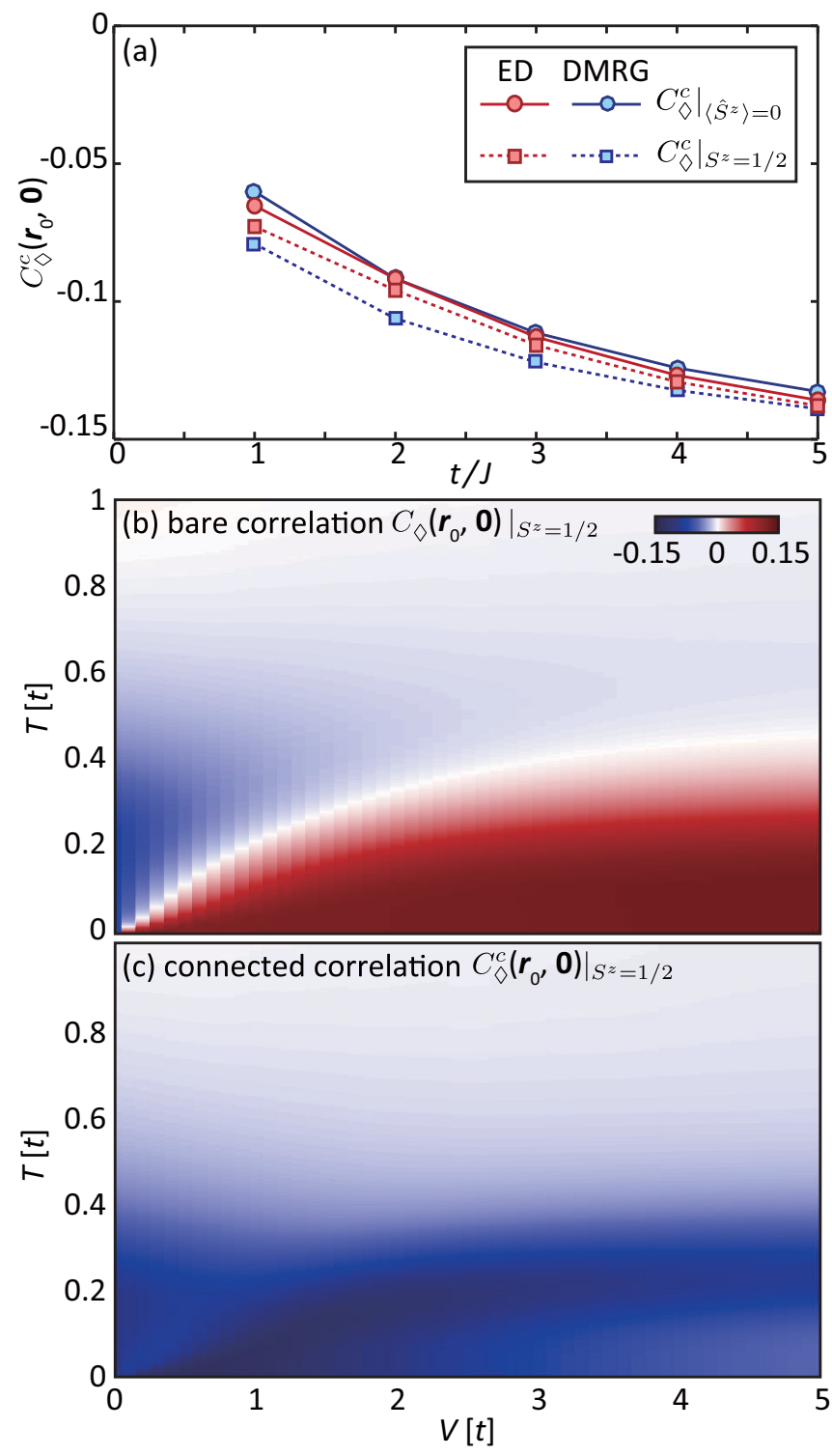

FIG. 7. (a) Genuine fifth-order correlator $C_{\diamond}^{c}$ for different spinensembles, $\left\langle\hat{S}^{z}\right\rangle=0$ and $S^{z}= \pm 1 / 2$, at zero temperature and without pinning potential. The results are obtained by the ED (red) and DMRG (blue) simulations on a $6 \times 12$ cylinder and a $4 \times 4$ cluster respectively. (b) The bare fifth-order correlator $C_{\diamond}$ and (c) the genuine correlator $C_{\diamond}^{c}$ in a spin-imbalanced ensemble with fixed total $S^{z}=1 / 2$, as a function of pinning potential $V$ and temperature $T$. We consider a $t-J-3 s$ Hamiltonian.

bles, the genuine higher-order terms can differ significantly for different ensembles, as we find in Fig. 7(b).

Finally, to further illustrate the differences between spinbalanced and imbalanced ensembles, we consider a strongly simplified physical setting at zero temperature. We assume that the hole is fully pinned in the center of the system $(V \gg t)$ and take into account only Ising couplings $\sim J^{z} \hat{S}_{\mathbf{i}}^{z} \hat{S}_{\mathbf{j}}^{z}$ between the spins. The ground state of this toy model in the spin-imbalanced ensemble with $S^{z}=1 / 2$ is a classical Néel state $|\mathrm{N},+\rangle$ where a missing down-spin at $\mathbf{r}$ defines the pinned hole. Similarly, the ground state in the spin-imbalanced ensemble with $S^{z}=-1 / 2$ is the 
opposite Néel state $|\mathrm{N},-\rangle$, obtained from $|\mathrm{N},+\rangle$ by flipping all spins. For both Néel states, the bare ring-spin correlator $\left.C_{\diamond}(\mathbf{r}, \mathbf{0})\right|_{S^{z}=1 / 2}=\left.C_{\diamond}(\mathbf{r}, \mathbf{0})\right|_{S^{z}=-1 / 2}$ is equal since all four spins surrounding the hole are aligned. Hence, $\left.C_{\diamond}(\mathbf{r}, \mathbf{0})\right|_{\left\langle\hat{S}^{z}\right\rangle=0}=$ $\left.C_{\diamond}(\mathbf{r}, \mathbf{0})\right|_{S^{z}= \pm 1 / 2}$ also takes the same value in the spinbalanced ensemble, defined by the even statistical mixture $\{|\mathrm{N},+\rangle,|\mathrm{N},-\rangle\}$. Now we turn to the connected correlators. For each of the two Néel states $|\mathrm{N}, \pm\rangle$ taken individually, $\left.C_{\diamond}^{c}\right|_{S^{z}= \pm 1 / 2}=0$ vanishes: By construction, the higher-order connected correlators are identically zero for product states. To obtain this result from Eq. (10), it is important to account for all lower-order contributions, including those with an odd number of $\hat{S}^{z}$ operators. In contrast, in the spin-balanced ensemble where $\left\langle\hat{S}^{z}\right\rangle=0$, only lower-order terms with an even number of $\hat{S}^{z}$ operators contribute in Eq. (10). This leads to a strongly modified connected correlator, with an overall negative value: indeed, counting all terms for the classical Néel states gives the estimate $\left.C_{\diamond}^{c}(\mathbf{r}, \mathbf{0})\right|_{\left\langle\hat{S}^{z}\right\rangle=0}=-2$ in our toy model with Ising interactions.

\section{DISCUSSIONS AND CONCLUSIONS}

Using the third-order and fifth-order spin-hole correlators, we have analyzed spatial correlation of a doped hole and its surrounding spin fluctuations in the single-hole doped Hubbard and $t-J-3 s$ models. We particularly investigate the impact of impurity on these high-order correlations, mimicked by a localized pinning potential with varying strength $V$. Interestingly, we find that even an extremely local impurity imposes an outsized effect on the high-order correlations. With the increase of the pinning potential, we identified a crossover from screening to antiscreening of the spin fluctuations surrounding the hole: For a mobile hole, the weakened third-order correlators in proximity to the dopant and the negative fifth-order ring-spin correlators reflect the underlying spin polaron formation-here the motion of the hole screens the spin defects; for an immobile hole trapped by the pinning potential, the third-order correlators in proximity to the dopant are enhanced and the bare fifth-order ring-spin correlators are positive - as a result of the antiscreened magnetic moment of the pinned hole. In this case, a geometric defect and a staggered field are induced in the AFM background. This microscopic transition from a spin-polaron to an antiscreened defect provides a route to understand the Zn-substitution experiments, where the magnetic moment is enhanced while superconductivity is suppressed. Due to the breaking of the spin-polaron, different holes are no longer paired by sharing the spin fluctuations. Instead, the locally enhanced spin fluctuations surrounding, the impurity-trapped hole, indicates a repulsion to other holes and therefore suppresses the coherence of Cooper pairs. This crossover in high-order correlators may also provide intuitions to impurity effects in other correlated materials.

We have also examined the impact of temperature on the considered spin-charge correlations. We find that thermal fluctuations lead to an extended spin-polaron regime with a weakly pinned hole, and identify the crossover to the pinned hole as a function of both temperature $T$ and pinning potential $V$. In contrast to the crossover, we found genuine (i.e., connected) fifth-order ring-spin correlators carrying differ- ent underlying physics: they are sizably negative and robust against the pinning potential.

Based on these observations, we demonstrated that higherorder correlation functions provide a new perspective on quantum many-body systems with strong correlations. This is a particularly promising direction in the context of correlated fermionic systems and quantum materials, where traditional theoretical approaches are limited and a comprehensive physical picture is still lacking. The specific situation discussed here, where we analyzed the effect of a localized pinning potential on mobile dopants, is particularly relevant in the solid-state context for understanding the effects of disorder on strong spin-charge correlations [56-63]. More generally, recent high-precision spectroscopic measurements in correlated materials have shown signatures of nonsymmetrybreaking gapped states, which are highly fluctuating and beyond the description of the low-order Gaussian wave functions [121-124]. The underlying physics of these phases lies in the non-Gaussian wave functions. The high-order correlations provide an efficient route to quantify these non-Gaussian features, as the Gaussian wave functions fractionalize all correlations two two-point ones through Wick's theorem. Although wave function itself is high-dimensional and cannot be read from experiments, these higher-order correlators, carrying the non-Gaussian features, can be directly measured and tested in state-of-the-art quantum gas microscopy experiments with ultracold atoms in optical lattices. The required temperatures to observe nontrivial effects have already been reached, and we expect that even lower temperatures will become accessible in the near future.

\section{ACKNOWLEDGMENTS}

Y.W. acknowledges support from the National Science Foundation (NSF) award DMR-2038011. A.B. and F.G. acknowledge support from Deutsche Forschungsgemeinschaft (DFG, German Research Foundation) under Germany's Excellence Strategy-EXC-2111-390814868. J.K. acknowledges funding from Hector Fellow Academy and support from the Max Planck Harvard Research Center for Quantum Optics (MPHQ). E.D. acknowledges support from Harvard-MIT CUA, ARO Grant No. W911NF-20-1-0163, the NSF award OAC-193471, and Harvard Quantum Initiative. This research used resources of the National Energy Research Scientific Computing Center (NERSC), a U.S. Department of Energy Office of Science User Facility operated under Contract No. DE-AC02-05CH11231. A.B. acknowledges the NSF through a grant for the Institute for Theoretical Atomic, Molecular, and Optical Physics at Harvard University and the Smithsonian Astrophysical Observatory.

\section{APPENDIX A: DETAILS ABOUT THE DIFFERENTIAL THIRD-ORDER CORRELATOR}

The differential third-order correlator has been briefly discussed in Sec. III. To reveal the impact of temperature and the pinning potential, we present its $V-T$ dependence of $\Delta B_{\mathrm{nn}}$ in Fig. 8, following the same manner of Fig. 3(c) in the main text. 


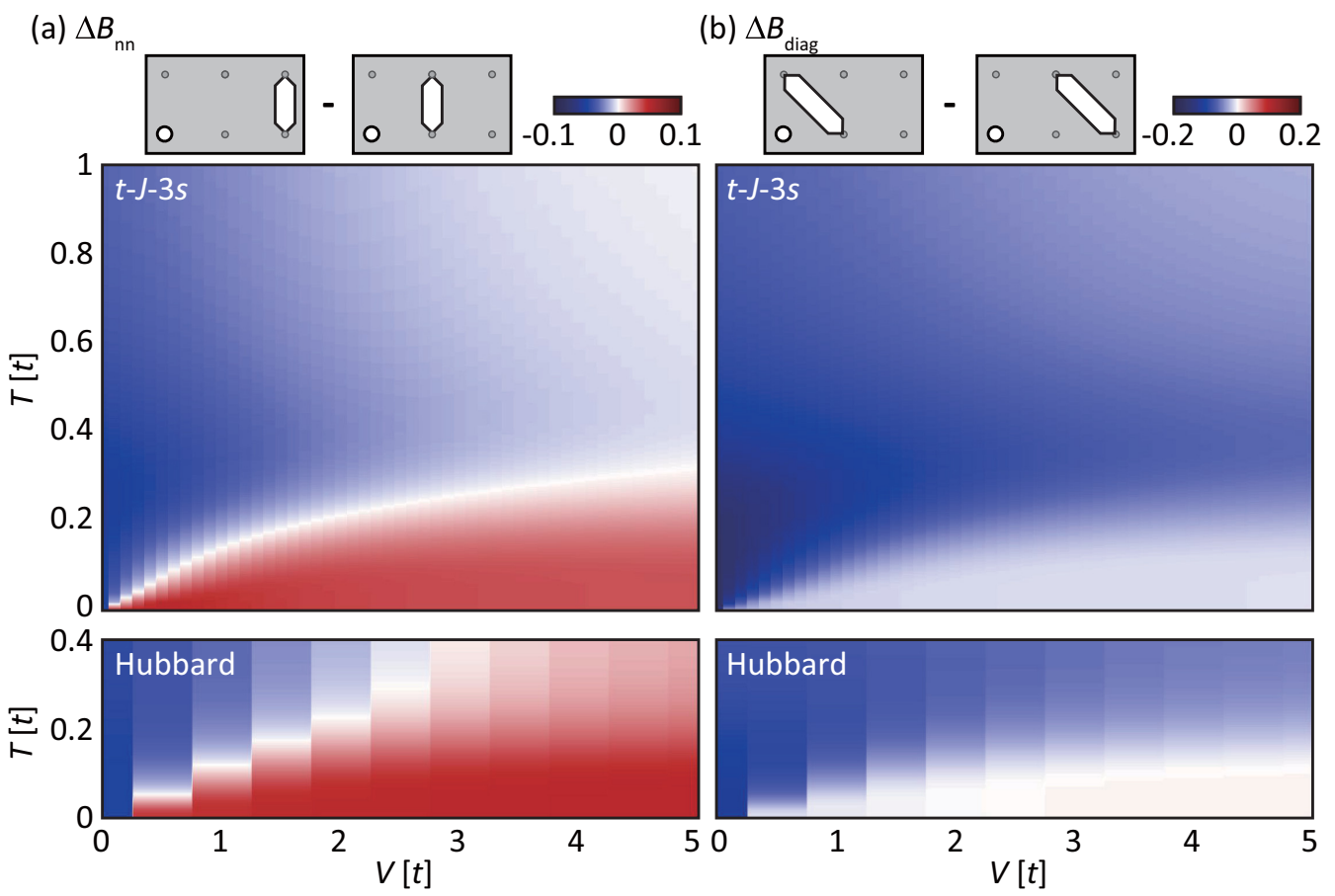

FIG. 8. (a) Dependence of the differential third-order correlators $\Delta B_{\mathrm{nn}}$ with bond lengths $|\mathbf{d}|=1$ as a function of the trapping potential $V$ and temperature $T$, obtained for the $t-J-3 s$ model (top) and the Hubbard model (bottom). (b) The same as panel (a) but for $\Delta B_{\text {diag }}$ with $|\mathbf{d}|=\sqrt{2}$. The insets in panels (a) and (b) explain the definition of the differential correlators.

In addition to the $\Delta B_{\mathrm{nn}}$ defined in Eq. (7), we further quantify the difference between the diagonal (hole-spin) correlators as

$$
\Delta B_{\text {diag }}=B(\mathbf{r}, \mathbf{r}+\hat{x}, \hat{y}-\hat{x})-B(\mathbf{r}, \mathbf{r}+2 \hat{x}, \hat{y}-\hat{x}) .
$$

The spatial relations of both differences are illustrated in the inset of Fig. 8. Note, to compensate for the negative sign induced by the AFM sublattices, we swapped the order while defining these two differences.

We notice a difference between the $\Delta B_{\mathrm{nn}}$ [Fig. 8(a)] and the $\Delta B_{\text {diag }}$ [Fig. 8(b)]: although the screening effect has been dramatically suppressed for large pinning potentials following the similar crossover, $\Delta B_{\text {diag }}$ does not exhibit an evident sign change. Since spin correlations are inhomogeneous when the system breaks translational symmetry regardless of the hole, the differential correlator $\Delta B_{\text {diag }}$ has been affected by this background inhomogeneity, reducing the signal-to-noise ratio for describing the underlying properties of the spin-hole composite.

The advantage of the genuine correlator $B^{c}\left(\mathbf{r}, \mathbf{r}^{\prime}, \mathbf{d}\right)$ over the differential correlator $\Delta B_{\text {diag }}$ is reflected by comparing Figs. 3(c) and 8(b). Although both describe the diagonal spin correlations relative to the hole, the genuine correlator clearly separates the spin polaron (where it is sizeably negative) and the antiscreening regime (where it is sizeably positive). Especially in the large $V$ limit, the genuine correlator characterizes a narrower crossover compared with Fig. 3(a), due to the exclusion of noise in lower-order correlators.

\section{APPENDIX B: FINITE-SIZE SCALING}

To test the size dependence of these different types of highorder correlations, we further perform the DQMC simulations and extrapolate them into different system sizes. DQMC is an unbiased stochastic method based on the sampling of the auxiliary Hubbard-Stratonovich field. Due to the presence of the fermion sign problem, DQMC does not work for very low temperatures in a doped Hubbard model. However, at high temperatures, it can be extended to systems much larger than the accessibility of ED. Here, we used DQMC to benchmark the finite-size effect and the high-temperature behavior of these high-order correlations.

As shown in Fig. 9, the results obtained by ED and DQMC agree well for $T<0.4 t$, above which the ED results are no longer reliable within the same number of excited states. A slightly larger deviation occurs for the connected fifth-order correlation $C_{\diamond}^{c}$. This can be attributed to two reasons. On the one hand, the DQMC simulation is based on grand canonical ensemble with a fixed average electron density instead of the canonical ensemble. The density fluctuation increases evidently with temperature, which is amplified in the high-order correlations. On the other hand, the excited-state truncation in ED leads to numerical errors at high-order correlations even when the static and low-order ones are numerically stable. Except for this slight deviation close to $0.4 t$, the results from both methods agree well.

With the increase of system size, the quantitative numbers change due to the dilution of the hole concentration (and also affected by the density fluctuation in the grand 

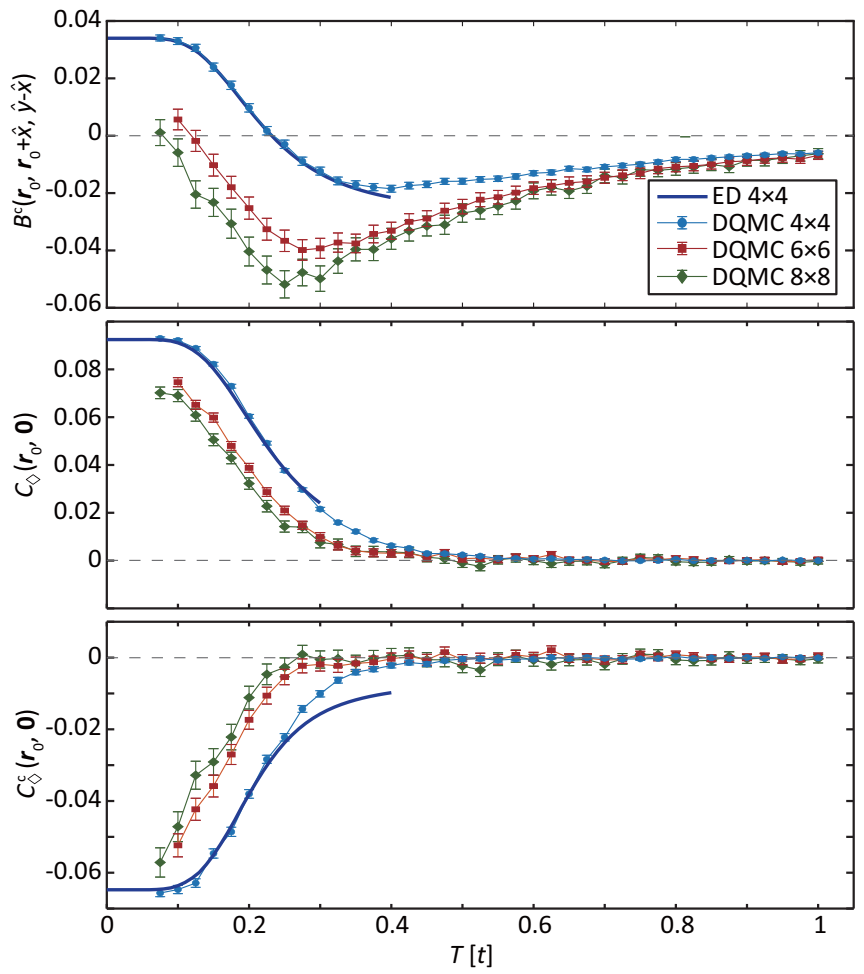

FIG. 9. Upper to lower: Temperature dependence of $B^{c}$ and $C_{\diamond}^{c}$ for the Hubbard model with pinning potential $V=5 t$. The blue, red, and green dots represent the DQMC results for $4 \times 4,6 \times 6$, and $8 \times$ 8 systems, while the thick blue curve represents the ED results for the $4 \times 4$ cluster. The DQMC results are truncated at low temperatures due to the fermion sign problem, while the ED results are restricted to $T<0.4 t$ due to the finite excited states kept in the calculation.

[1] E. Dagotto, Correlated electrons in high-temperature superconductors, Rev. Mod. Phys. 66, 763 (1994).

[2] P. W. Anderson, The resonating valence bond state in $\mathrm{La}_{2} \mathrm{CuO}_{4}$ and superconductivity, Science 235, 1196 (1987).

[3] F. C. Zhang and T. M. Rice, Effective Hamiltonian for the superconducting Cu oxides, Phys. Rev. B 37, 3759 (1988).

[4] P. A. Lee, N. Nagaosa, and X.-G. Wen, Doping a Mott insulator: Physics of high-temperature superconductivity, Rev. Mod. Phys. 78, 17 (2006).

[5] A. Singh and P. Goswami, Spin-wave spectrum in $\mathrm{La}_{2} \mathrm{CuO}_{4}$ : Double occupancy and competing interaction effects, Phys. Rev. B 66, 092402 (2002).

[6] B.-X. Zheng, C.-M. Chung, P. Corboz, G. Ehlers, M.-P. Qin, R. M. Noack, H. Shi, S. R. White, S. Zhang, and G. K.-L. Chan, Stripe order in the underdoped region of the twodimensional Hubbard model, Science 358, 1155 (2017).

[7] E. W. Huang, C. B. Mendl, S. Liu, S. Johnston, H.-C. Jiang, B. Moritz, and T. P. Devereaux, Numerical evidence of fluctuating stripes in the normal state of high- $T_{c}$ cuprate superconductors, Science 358, 1161 (2017).

[8] B. Ponsioen, S. S. Chung, and P. Corboz, Period 4 stripe in the extended two-dimensional Hubbard model, Phys. Rev. B 100, 195141 (2019).

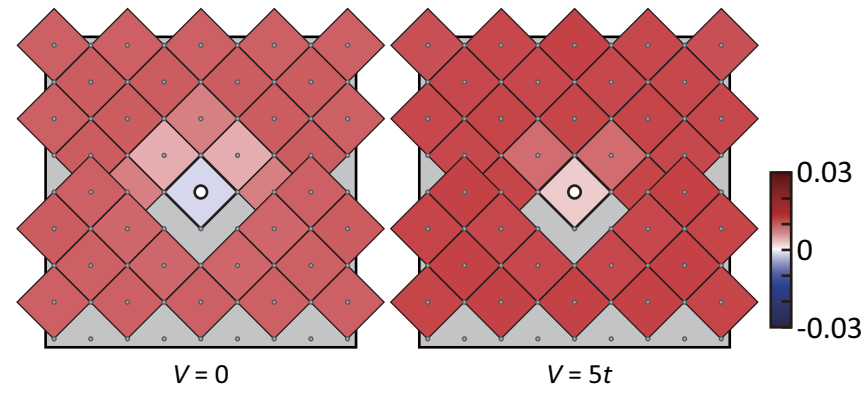

FIG. 10. The spatial distribution of $C_{\diamond}(\mathbf{r}, \mathbf{d})$ calculated using DQMC for a $8 \times 8$ cluster with $T=0.4 t$. The left and right panel show the results without and with a pinning potential, respectively.

canonical ensemble). However, the sign change in $B^{c}$ is preserved, reflecting the crossover between the screening and antiscreening picture. Due to the strong density fluctuation at $V=0$, DQMC is not suitable for discussing $V$-dependence in these high-order correlations. The crossover for different pinning potentials at zero temperature has been benchmarked in larger systems using DMRG in Fig. 5(a) and Ref. [113].

Since our DQMC simulations cannot reach a low enough temperature for the $8 \times 8$ cluster, the crossover of the fifthorder correlator $C_{\diamond}$ cannot be revealed by tuning temperature. To show this crossover from a different perspective, we select $T=0.4 t$ and calculate the spatial distribution of $C_{\diamond}(\mathbf{r}, \mathbf{d})$ for different pinning potentials in the same manner of Fig. 5. As shown in Fig. 10, the local fifth-order correlator $C_{\diamond}\left(\mathbf{r}_{0}, \mathbf{0}\right)$ suffered from a sign change from negative at $V=0$ to positive at $V=5 t$, while other correlators at longer distances do not exhibit obvious changes. This phenomenon is consistent with the crossover extracted from ED at small clusters.
[9] J. Kokalj, Bad-metallic behavior of doped Mott insulators, Phys. Rev. B 95, 041110(R) (2017).

[10] E. W. Huang, R. Sheppard, B. Moritz, and T. P. Devereaux, Strange metallicity in the doped Hubbard model, Science 366, 987 (2019).

[11] P. Cha, A. A. Patel, E. Gull, and E.-A. Kim, Slope invariant $T$-linear resistivity from local self-energy, Phys. Rev. Res. 2, 033434 (2020).

[12] T. A. Maier, M. Jarrell, T. C. Schulthess, P. R. C. Kent, and J. B. White, Systematic Study of $d$-Wave Superconductivity in the 2D Repulsive Hubbard Model, Phys. Rev. Lett. 95, 237001 (2005)

[13] B.-X. Zheng and Gamet Kin-Lic Chan, Ground-state phase diagram of the square lattice Hubbard model from density matrix embedding theory, Phys. Rev. B 93, 035126 (2016).

[14] K. Ido, T. Ohgoe, and M. Imada, Competition among various charge-inhomogeneous states and $d$-wave superconducting state in Hubbard models on square lattices, Phys. Rev. B 97, 045138 (2018).

[15] H.-C. Jiang and T. P. Devereaux, Superconductivity in the doped Hubbard model and its interplay with next-nearest hopping $t^{\prime}$, Science 365, 1424 (2019). 
[16] C.-M. Chung, M. Qin, S. Zhang, U. Schollwöck, and S. R. White, Plaquette versus ordinary $d$-wave pairing in the $t^{\prime}$-Hubbard model on a width-4 cylinder, Phys. Rev. B 102, 041106(R) (2020).

[17] Y. Ohta, T. Tohyama, and S. Maekawa, Apex oxygen and critical temperature in copper oxide superconductors: Universal correlation with the stability of local singlets, Phys. Rev. B 43, 2968 (1991).

[18] A. Lanzara, P. Bogdanov, X. Zhou, S. Kellar, D. Feng, E. Lu, T. Yoshida, H. Eisaki, A. Fujimori, K. Kishio et al., Evidence for ubiquitous strong electron-phonon coupling in high-temperature superconductors, Nature (London) 412, 510 (2001).

[19] K. M. Shen, F. Ronning, D. H. Lu, W. S. Lee, N. J. C. Ingle, W. Meevasana, F. Baumberger, A. Damascelli, N. P. Armitage, L. L. Miller, Y. Kohsaka, M. Azuma, M. Takano, H. Takagi, and Z. X. Shen, Missing Quasiparticles and the Chemical Potential Puzzle in the Doping Evolution of the Cuprate Superconductors, Phys. Rev. Lett. 93, 267002 (2004).

[20] A. S. Mishchenko and N. Nagaosa, Electron-Phonon Coupling and a Polaron in the $t-J$ Model: From the Weak to the Strong Coupling Regime, Phys. Rev. Lett. 93, 036402 (2004).

[21] T. Cuk, F. Baumberger, D. H. Lu, N. Ingle, X. J. Zhou, H. Eisaki, N. Kaneko, Z. Hussain, T. P. Devereaux, N. Nagaosa, and Z. X. Shen, Coupling of the $\mathrm{B}_{1 g}$ Phonon to the Antinodal Electronic States of $\mathrm{Bi}_{2} \mathrm{Sr}_{2} \mathrm{Ca}_{0.92} \mathrm{Y}_{0.08} \mathrm{Cu}_{2} \mathrm{O}_{8+\delta}$, Phys. Rev. Lett. 93, 117003 (2004).

[22] D. Reznik, L. Pintschovius, M. Ito, S. Iikubo, M. Sato, H. Goka, M. Fujita, K. Yamada, G. Gu, and J. Tranquada, Electron-phonon coupling reflecting dynamic charge inhomogeneity in copper oxide superconductors, Nature (London) 440, 1170 (2006).

[23] S. Johnston, I. M. Vishik, W. S. Lee, F. Schmitt, S. Uchida, K. Fujita, S. Ishida, N. Nagaosa, Z. X. Shen, and T. P. Devereaux, Evidence for the Importance of Extended Coulomb Interactions and Forward Scattering in Cuprate Superconductors, Phys. Rev. Lett. 108, 166404 (2012).

[24] Y. Peng, G. Dellea, M. Minola, M. Conni, A. Amorese, D. Di Castro, G. De Luca, K. Kummer, M. Salluzzo, X. Sun et al., Influence of apical oxygen on the extent of in-plane exchange interaction in cuprate superconductors, Nat. Phys. 13, 1201 (2017).

[25] Y. He, M. Hashimoto, D. Song, S.-D. Chen, J. He, I. Vishik, B. Moritz, D.-H. Lee, N. Nagaosa, J. Zaanen et al., Rapid change of superconductivity and electron-phonon coupling through critical doping in Bi-2212, Science 362, 62 (2018).

[26] E. Demler, W. Hanke, and S.-C. Zhang, SO(5) theory of antiferromagnetism and superconductivity, Rev. Mod. Phys. 76, 909 (2004).

[27] D. J. Scalapino, A common thread: The pairing interaction for unconventional superconductors, Rev. Mod. Phys. 84, 1383 (2012).

[28] T. A. Maier, P. Staar, V. Mishra, U. Chatterjee, J. C. Campuzano, and D. J. Scalapino, Pairing in a dry Fermi sea, Nat. Commun. 7, 11875 (2016).

[29] J. P. F. LeBlanc, A. E. Antipov, F. Becca, I. W. Bulik, G. K.-L. Chan, C. M. Chung, Y. Deng, M. Ferrero, T. M. Henderson, C. A. Jimenez-Hoyos, E. Kozik, X. W. Liu, A. J. Millis, N. V. Prokofev, M. Qin, G. E. Scuseria, H. Shi, B. V. Svistunov,
L. F. Tocchio, I. S. Tupitsyn, S. R. White, S. Zhang, B. X. Zheng, Z. Zhu, and E. Gull, Solutions of the Two-Dimensional Hubbard Model: Benchmarks and Results from a Wide Range of Numerical Algorithms, Phys. Rev. X 5, 041041 (2015).

[30] C. L. Kane, P. A. Lee, and N. Read, Motion of a single hole in a quantum antiferromagnet, Phys. Rev. B 39, 6880 (1989).

[31] S. Sachdev, Hole motion in a quantum Néel state, Phys. Rev. B 39, 12232 (1989).

[32] Z. Liu and E. Manousakis, Spectral function of a hole in the $t$-J model, Phys. Rev. B 44, 2414 (1991).

[33] G. Martinez and P. Horsch, Spin polarons in the $t$ - $J$ model, Phys. Rev. B 44, 317 (1991).

[34] W. Stephan and P. Horsch, Fermi Surface and Dynamics of the $t$ - $J$ Model at Moderate Doping, Phys. Rev. Lett. 66, 2258 (1991).

[35] E. Manousakis, The spin- $\frac{1}{2}$ Heisenberg antiferromagnet on a square lattice and its application to the cuprous oxides, Rev. Mod. Phys. 63, 1 (1991).

[36] E. Dagotto, A. Moreo, F. Ortolani, D. Poilblanc, and J. Riera, Static and dynamical properties of doped Hubbard clusters, Phys. Rev. B 45, 10741 (1992).

[37] Z. Liu and E. Manousakis, Dynamical properties of a hole in a Heisenberg antiferromagnet, Phys. Rev. B 45, 2425 (1992).

[38] W. Stephan and P. Horsch, Single-particle and optical excitations in doped Mott-Hubbard insulators, Int. J. Mod. Phys. B 6, 589 (1992).

[39] J. Bała, A. M. Oleś, and J. Zaanen, Spin polarons in the $t-t^{\prime}-J$ model, Phys. Rev. B 52, 4597 (1995).

[40] M. Brunner, F. F. Assaad, and A. Muramatsu, Single-hole dynamics in the $t-J$ model on a square lattice, Phys. Rev. B 62, 15480 (2000).

[41] D. J. Scalapino, E. Loh Jr., and J. E. Hirsch, $d$-wave pairing near a spin-density-wave instability, Phys. Rev. B 34, 8190 (1986).

[42] C. Gros, R. Joynt, and T. Rice, Superconducting instability in the large- $U$ limit of the two-dimensional Hubbard model, Z. Phys. B 68, 425 (1987).

[43] G. Kotliar and J. Liu, Superexchange mechanism and $d$-wave superconductivity, Phys. Rev. B 38, 5142 (1988).

[44] J. R. Schrieffer, X. G. Wen, and S. C. Zhang, Dynamic spin fluctuations and the bag mechanism of high- $T_{c}$ superconductivity, Phys. Rev. B 39, 11663 (1989).

[45] D. J. Scalapino, The case for $d_{x}^{2}-y^{2}$ pairing in the cuprate superconductors, Phys. Rep. 250, 329 (1995).

[46] C. Tsuei and J. Kirtley, Pairing symmetry in cuprate superconductors, Rev. Mod. Phys. 72, 969 (2000).

[47] J. Graf, G.-H. Gweon, K. McElroy, S. Y. Zhou, C. Jozwiak, E. Rotenberg, A. Bill, T. Sasagawa, H. Eisaki, S. Uchida, H. Takagi, D. H Lee, and A. Lanzara, Universal High Energy Anomaly in the Angle-Resolved Photoemission Spectra of High Temperature Superconductors: Possible Evidence of Spinon and Holon Branches, Phys. Rev. Lett. 98, 067004 (2007).

[48] J. He, C. R. Rotundu, M. S. Scheurer, Y. He, M. Hashimoto, K.-J. Xu, Y. Wang, E. W. Huang, T. Jia, S. Chen et al., Fermi surface reconstruction in electron-doped cuprates without antiferromagnetic long-range order, Proc. Natl. Acad. Sci. USA 116, 3449 (2019). 
[49] S.-D. Chen, M. Hashimoto, Y. He, D. Song, K.-J. Xu, J.-F. He, T. P. Devereaux, H. Eisaki, D.-H. Lu, J. Zaanen et al., Incoherent strange metal sharply bounded by a critical doping in Bi2212, Science 366, 1099 (2019).

[50] M. Le Tacon, G. Ghiringhelli, J. Chaloupka, M. M. Sala, V. Hinkov, M. Haverkort, M. Minola, M. Bakr, K. Zhou, S. Blanco-Canosa et al., Intense paramagnon excitations in a large family of high-temperature superconductors, Nat. Phys. 7, 725 (2011).

[51] M. Dean, G. Dellea, R. Springell, F. Yakhou-Harris, K. Kummer, N. Brookes, X. Liu, Y. Sun, J. Strle, T. Schmitt et al., Persistence of magnetic excitations in $\mathrm{La}_{2-x} \mathrm{Sr}_{x} \mathrm{CuO}_{4}$ from the undoped insulator to the heavily overdoped nonsuperconducting metal, Nat. Mater. 12, 1019 (2013).

[52] M. P. M. Dean, A. J. A. James, R. S. Springell, X. Liu, C. Monney, K. J. Zhou, R. M. Konik, J. S. Wen, Z. J. Xu, G. D. Gu, V. N. Strocov, T. Schmitt, and J. P. Hill, HighEnergy Magnetic Excitations in the Cuprate Superconductor $\mathrm{Bi}_{2} \mathrm{Sr}_{2} \mathrm{CaCu}_{2} \mathrm{O}_{8+\delta}$ : Towards a Unified Description of its Electronic and Magnetic Degrees of Freedom, Phys. Rev. Lett. 110, 147001 (2013).

[53] W. S. Lee, J. J. Lee, E. A. Nowadnick, S. Gerber, W. Tabis, S. W. Huang, V. N. Strocov, E. M. Motoyama, G. Yu, B. Moritz et al., Asymmetry of collective excitations in electron-and hole-doped cuprate superconductors, Nat. Phys. 10, 883 (2014).

[54] K. Ishii, M. Fujita, T. Sasaki, M. Minola, G. Dellea, C. Mazzoli, K. Kummer, G. Ghiringhelli, L. Braicovich, T. Tohyama et al., High-energy spin and charge excitations in electron-doped copper oxide superconductors, Nat. Commun. 5, 3714 (2014).

[55] N. Hasselmann, A. H. Castro Neto, and C. M. Smith, Spinglass phase of cuprates, Phys. Rev. B 69, 014424 (2004).

[56] A. V. Mahajan, H. Alloul, G. Collin, and J. F. Marucco, ${ }^{89} \mathrm{Y}$ NMR Probe of $\mathrm{Zn}$ Induced Local Magnetism in $\mathrm{YBa}_{2}\left(\mathrm{Cu}_{1-y} \mathrm{Zn}_{y}\right)_{3} \mathrm{O}_{6+x}$, Phys. Rev. Lett. 72, 3100 (1994).

[57] H. Alloul, P. Mendels, H. Casalta, J. Marucco, and J. F. Arabski, Correlations between Magnetic and Superconducting Properties of $\mathrm{Zn}$-Substituted $\mathrm{YBa}_{2} \mathrm{Cu}_{3} \mathrm{O}_{6+x}$, Phys. Rev. Lett. 67, 3140 (1991).

[58] G. Xiao, M. Z. Cieplak, J. Q. Xiao, and C. L. Chien, Magnetic pair-breaking effects: Moment formation and critical doping level in superconducting $\mathrm{La}_{1.85} \mathrm{Sr}_{0.15} \mathrm{Cu}_{1-x} A_{x} \mathrm{O}_{4}$ systems ( $A=\mathrm{Fe}, \mathrm{Co}, \mathrm{Ni}, \mathrm{Zn}, \mathrm{Ga}, \mathrm{Al}$ ), Phys. Rev. B 42, 8752 (1990).

[59] P. Mendels, H. Alloul, J. H. Brewer, G. D. Morris, T. L. Duty, S. Johnston, E. J. Ansaldo, G. Collin, J. F. Marucco, C. Niedermayer, D. R. Noakes, and C. E. Stronach, Muon-spinrotation study of the effect of $\mathrm{Zn}$ substitution on magnetism in $\mathrm{YBa}_{2} \mathrm{Cu}_{3} \mathrm{O}_{x}$, Phys. Rev. B 49, 10035 (1994).

[60] A. Maeda, T. Yabe, S. Takebayashi, M. Hase, and K. Uchinokura, Substitution of $3 d$ metals for $\mathrm{Cu}$ in $\mathrm{Bi}_{2}\left(\mathrm{Sr}_{0.6} \mathrm{Ca}_{0.4}\right)_{3} \mathrm{Cu}_{2} \mathrm{O}_{y}$, Phys. Rev. B 41, 4112 (1990).

[61] Y. Fukuzumi, K. Mizuhashi, K. Takenaka, and S. Uchida, Universal Superconductor-Insulator Transition and $T_{c}$ Depression in Zn-substituted high- $T_{c}$ Cuprates in the Underdoped Regime, Phys. Rev. Lett. 76, 684 (1996).

[62] B. Nachumi, A. Keren, K. Kojima, M. Larkin, G. M. Luke, J. Merrin, O. Tchernyshov, Y. J. Uemura, N. Ichikawa, M. Goto, and S. Uchida, Muon Spin Relaxation Studies of
Zn-Substitution Effects in High- $T_{c}$ Cuprate Superconductors, Phys. Rev. Lett. 77, 5421 (1996).

[63] D. N. Basov, B. Dabrowski, and T. Timusk, Infrared Probe of Transition from Superconductor to Nonmetal in $\mathrm{YBa}_{2}\left(\mathrm{Cu}_{1-x} \mathrm{Zn}_{x}\right)_{4} \mathrm{O}_{8}$, Phys. Rev. Lett. 81, 2132 (1998).

[64] P. A. Lee, Localized States in a $d$-Wave Superconductor, Phys. Rev. Lett. 71, 1887 (1993).

[65] Y. Sun and K. Maki, Impurity effects in $d$-wave superconductors, Phys. Rev. B 51, 6059 (1995).

[66] M. I. Salkola, A. V. Balatsky, and D. J. Scalapino, Theory of Scanning Tunneling Microscopy Probe of Impurity States in a $d$-Wave Superconductor, Phys. Rev. Lett. 77, 1841 (1996).

[67] P. J. Hirschfeld and W. O. Putikka, Theory of Thermal Conductivity in $\mathrm{YBa}_{2} \mathrm{Cu}_{3} \mathrm{O}_{7-\delta}$, Phys. Rev. Lett. 77, 3909 (1996).

[68] M. Franz, C. Kallin, and A. J. Berlinsky, Impurity scattering and localization in $d$-wave superconductors, Phys. Rev. B 54, R6897 (1996).

[69] H. Tsuchiura, Y. Tanaka, M. Ogata, and S. Kashiwaya, Quasiparticle properties around a nonmagnetic impurity in the superconducting state of the two-dimensional $t$ - $J$ model, J. Phys. Soc. Jpn. 68, 2510 (1999).

[70] S. A. Kivelson, E. Fradkin, and V. J. Emery, Electronic liquidcrystal phases of a doped Mott insulator, Nature (London) 393, 550 (1998).

[71] V. Emery, S. Kivelson, and J. Tranquada, Stripe phases in high-temperature superconductors, Proc. Nat. Acad. Sci. USA 96, 8814 (1999).

[72] S. A. Kivelson, I. P. Bindloss, E. Fradkin, V. Oganesyan, J. Tranquada, A. Kapitulnik, and C. Howald, How to detect fluctuating stripes in the high-temperature superconductors, Rev. Mod. Phys. 75, 1201 (2003).

[73] C. Gross and I. Bloch, Quantum simulations with ultracold atoms in optical lattices, Science 357, 995 (2017).

[74] W. S. Bakr, J. I. Gillen, A. Peng, S. Fölling, and M. Greiner, A quantum gas microscope for detecting single atoms in a Hubbard-regime optical lattice, Nature (London) 462, 74 (2009).

[75] J. F. Sherson, C. Weitenberg, M. Endres, M. Cheneau, I. Bloch, and S. Kuhr, Single-atom-resolved fluorescence imaging of an atomic Mott insulator, Nature (London) 467, 68 (2010).

[76] M. F. Parsons, F. Huber, A. Mazurenko, C. S. Chiu, W. Setiawan, K. Wooley-Brown, S. Blatt, and M. Greiner, SiteResolved Imaging of Fermionic $\mathrm{Li}_{6}$ in an Optical Lattice, Phys. Rev. Lett. 114, 213002 (2015).

[77] L. W. Cheuk, M. A. Nichols, M. Okan, T. Gersdorf, V. V. Ramasesh, W. S. Bakr, T. Lompe, and M. W. Zwierlein, Quantum-Gas Microscope for Fermionic Atoms, Phys. Rev. Lett. 114, 193001 (2015).

[78] A. Omran, M. Boll, T. A. Hilker, K. Kleinlein, G. Salomon, I. Bloch, and C. Gross, Microscopic Observation of Pauli Blocking in Degenerate Fermionic Lattice Gases, Phys. Rev. Lett. 115, 263001 (2015).

[79] G. J. A. Edge, R. Anderson, D. Jervis, D. C. McKay, R. Day, S. Trotzky, and J. H. Thywissen, Imaging and addressing of individual fermionic atoms in an optical lattice, Phys. Rev. A 92, 063406 (2015).

[80] E. Haller, J. Hudson, A. Kelly, D. A. Cotta, B. Peaudecerf, G. D. Bruce, and S. Kuhr, Single-atom imaging of fermions in a quantum-gas microscope, Nat. Phys. 11, 738 (2015). 
[81] A. Mazurenko, C. S. Chiu, G. Ji, M. F. Parsons, M. KanászNagy, R. Schmidt, F. Grusdt, E. Demler, D. Greif, and M. Greiner, A cold-atom Fermi-Hubbard antiferromagnet, Nature (London) 545, 462 (2017).

[82] T. A. Hilker, G. Salomon, F. Grusdt, A. Omran, M. Boll, E. Demler, I. Bloch, and C. Gross, Revealing hidden antiferromagnetic correlations in doped Hubbard chains via string correlators, Science 357, 484 (2017).

[83] C. S. Chiu, G. Ji, A. Bohrdt, M. Xu, M. Knap, E. Demler, F. Grusdt, M. Greiner, and D. Greif, String patterns in the doped Hubbard model, Science 365, 251 (2019).

[84] T. Schweigler, V. Kasper, S. Erne, I. Mazets, B. Rauer, F. Cataldini, T. Langen, T. Gasenzer, J. Berges, and J. Schmiedmayer, Experimental characterization of a quantum many-body system via higher-order correlations, Nature (London) 545, 323 (2017).

[85] G. Salomon, J. Koepsell, J. Vijayan, T. A. Hilker, J. Nespolo, L. Pollet, I. Bloch, and C. Gross, Direct observation of incommensurate magnetism in Hubbard chains, Nature (London) 565, 56 (2019).

[86] P. T. Brown, D. Mitra, E. Guardado-Sanchez, R. Nourafkan, A. Reymbaut, C.-D. Hébert, S. Bergeron, A.-M. Tremblay, J. Kokalj, D. A. Huse et al., Bad metallic transport in a cold atom Fermi-Hubbard system, Science 363, 379 (2019).

[87] M. A. Nichols, L. W. Cheuk, M. Okan, T. R. Hartke, E. Mendez, T. Senthil, E. Khatami, H. Zhang, and M. W. Zwierlein, Spin transport in a Mott insulator of ultracold fermions, Science 363, 383 (2019).

[88] J. Koepsell, J. Vijayan, P. Sompet, F. Grusdt, T. A. Hilker, E. Demler, G. Salomon, I. Bloch, and C. Gross, Imaging magnetic polarons in the doped Fermi-Hubbard model, Nature (London) 572, 358 (2019).

[89] A. Bohrdt, Y. Wang, J. Koepsell, M. Kánasz-Nagy, E. Demler, and F. Grusdt, Dominant Fifth-Order Correlations in Doped Quantum Antiferromagnets, Phys. Rev. Lett. 126, 026401 (2021).

[90] J. Koepsell, D. Bourgund, P. Sompet, S. Hirthe, A. Bohrdt, Y. Wang, F. Grusdt, E. Demler, G. Salomon, C. Gross et al., Microscopic evolution of doped Mott insulators from polaronic metal to Fermi liquid, arXiv:2009.04440.

[91] W. Hofstetter, J. I. Cirac, P. Zoller, E. Demler, and M. D. Lukin, High-Temperature Superfluidity of Fermionic Atoms in Optical Lattices, Phys. Rev. Lett. 89, 220407 (2002).

[92] A. F. Ho, M. A. Cazalilla, and T. Giamarchi, Quantum simulation of the Hubbard model: The attractive route, Phys. Rev. A 79, 033620 (2009).

[93] R. Jördens, L. Tarruell, D. Greif, T. Uehlinger, N. Strohmaier, H. Moritz, T. Esslinger, L. De Leo, C. Kollath, A. Georges et al., Quantitative Determination of Temperature in the Approach to Magnetic Order of Ultracold Frmions in an Optical Lattice, Phys. Rev. Lett. 104, 180401 (2010).

[94] T. Esslinger, Fermi-Hubbard physics with atoms in an optical lattice, Ann. Rev. Condens. Matter Phys. 1, 129 (2010).

[95] L. Tarruell and L. Sanchez-Palencia, Quantum simulation of the Hubbard model with ultracold fermions in optical lattices, C. R. Phys. 19, 365 (2018).

[96] K. Chao, J. Spalek, and A. Oleś, Kinetic exchange interaction in a narrow s-band, J. Phys. C: Solid State Phys 10, L271 (1977).
[97] K. A. Chao, J. Spałek, and A. M. Oleś, Canonical perturbation expansion of the Hubbard model, Phys. Rev. B 18, 3453 (1978).

[98] V. I. Belinicher and A. L. Chernyshev, Consistent low-energy reduction of the three-band model for copper oxides with OO hopping to the effective $t$ - $J$ model, Phys. Rev. B 49, 9746 (1994).

[99] V. I. Belinicher, A. L. Chernyshev, and L. V. Popovich, Range of the $t-J$ model parameters for $\mathrm{CuO}_{2}$ planes: Experimental data constraints, Phys. Rev. B 50, 13768 (1994).

[100] J. H. Jefferson, H. Eskes, and L. F. Feiner, Derivation of a single-band model for $\mathrm{CuO}_{2}$ planes by a cell-perturbation method, Phys. Rev. B 45, 7959 (1992).

[101] J. Spałek, Effect of pair hopping and magnitude of intraatomic interaction on exchange-mediated superconductivity, Phys. Rev. B 37, 533 (1988)

[102] K. J. von Szczepanski, P. Horsch, W. Stephan, and M. Ziegler, Single-particle excitations in a quantum antiferromagnet, Phys. Rev. B 41, 2017 (1990).

[103] H. Eskes and A. M. Oleś, Two Hubbard Bands: Weight Transfer in Optical and One-Particle Spectra, Phys. Rev. Lett. 73, 1279 (1994).

[104] H. Eskes, A. M. Oleś, M. B. J. Meinders, and W. Stephan, Spectral properties of the Hubbard bands, Phys. Rev. B 50, 17980 (1994).

[105] V. I. Belinicher, A. L. Chernyshev, and V. A. Shubin, Generalized $t-t^{\prime}-J$ model: Parameters and single-particle spectrum for electrons and holes in copper oxides, Phys. Rev. B 53, 335 (1996).

[106] V. I. Belinicher, A. L. Chernyshev, and V. A. Shubin, Singlehole dispersion relation for the real $\mathrm{CuO}_{2}$ plane, Phys. Rev. B 54, 14914 (1996).

[107] G. C. Psaltakis, Optical absorption in the canted antiferromagnetic phase of a $t-J$ model, Phys. Rev. B 45, 539 (1992).

[108] H. Eskes and R. Eder, Hubbard model versus $t$ - $J$ model: The one-particle spectrum, Phys. Rev. B 54, R14226 (1996).

[109] R. B. Lehoucq, D. C. Sorensen, and C. Yang, ARPACK Users' Guide: Solution of Large-Scale Eigenvalue Problems with Implicitly Restarted Arnoldi Methods (SIAM, Philadelphia, PA, 1998).

[110] C. Jia, Y. Wang, C. Mendl, B. Moritz, and T. Devereaux, Paradeisos: A perfect hashing algorithm for many-body eigenvalue problems, Comput. Phys. Commun. 224, 81 (2018).

[111] R. Blankenbecler, D. J. Scalapino, and R. L. Sugar, Monte Carlo calculations of coupled boson-fermion systems, Phys. Rev. D 24, 2278 (1981).

[112] S. R. White, D. J. Scalapino, R. L. Sugar, E. Y. Loh, J. E. Gubernatis, and R. T. Scalettar, Numerical study of the twodimensional Hubbard model, Phys. Rev. B 40, 506 (1989).

[113] F. Grusdt, A. Bohrdt, and E. Demler, Microscopic spinonchargon theory of magnetic polarons in the $t$ - $J$ model, Phys. Rev. B 99, 224422 (2019).

[114] E. Blomquist and J. Carlström, Unbiased description of magnetic polarons in a Mott insulator, Commun. Phys. 3, 172 (2020).

[115] F. H. L. Essler, V. E. Korepin, and K. Schoutens, Complete Solution of the One-Dimensional Hubbard Model, Phys. Rev. Lett. 67, 3848 (1991).

[116] L. Seabra, F. H. L. Essler, F. Pollmann, I. Schneider, and $T$. Veness, Real-time dynamics in the one- 
dimensional Hubbard model, Phys. Rev. B 90, 245127 (2014).

[117] The fact that the correlation switches sign for any infinitesimal $V$ results from the extra hypercubic symmetry in a $4 \times 4$ Hubbard model. This symmetry leads to a sixfold degeneracy for the ground state without $V$ and is broken for any finite $V$. The critical $V$ should lie at a small finite value at the thermodynamic limit, similar to the presented results at finite temperature.

[118] F. Grusdt, M. Kánasz-Nagy, A. Bohrdt, C. S. Chiu, G. Ji, M. Greiner, D. Greif, and E. Demler, Parton Theory of Magnetic Polarons: Mesonic Resonances and Signatures in Dynamics, Phys. Rev. X 8, 011046 (2018).

[119] M. Boll, T. A. Hilker, G. Salomon, A. Omran, J. Nespolo, L. Pollet, I. Bloch, and C. Gross, Spin-and density-resolved microscopy of antiferromagnetic correlations in Fermi-Hubbard chains, Science 353, 1257 (2016).

[120] J. Koepsell, S. Hirthe, D. Bourgund, P. Sompet, J. Vijayan, G. Salomon, C. Gross, and I. Bloch, Robust bilayer Charge-
Pumping for Spin-and Density-Resolved Quantum Gas Microscopy, Phys. Rev. Lett. 125, 010403 (2020).

[121] T. Kondo, W. Malaeb, Y. Ishida, T. Sasagawa, H. Sakamoto, T. Takeuchi, T. Tohyama, and S. Shin, Point nodes persisting far beyond $T_{c}$ in Bi2212, Nat. Commun. 6, 7699 (2015).

[122] Y. He, S.-D. Chen, Z.-X. Li, D. Zhao, D. Song, Y. Yoshida, H. Eisaki, T. Wu, X.-H. Chen, D.-H. Lu et al., Fluctuating superconductivity in overdoped cuprate with a flat antinodal dispersion, arXiv:2009.10932.

[123] B. D. Faeth, S.-L. Yang, J. K. Kawasaki, J. N. Nelson, P. Mishra, C. T. Parzyck, C. Li, D. G. Schlom, and K. M. Shen, Incoherent Cooper Pairing and Pseudogap Behavior in Single-Layer FeSe/SrTiO 3 , Phys. Rev. X 11, 021054 (2021).

[124] Y. Xu, H. Rong, Q. Wang, D. Wu, Y. Hu, Y. Cai, Q. Gao, H. Yan, C. Li, C. Yin et al., Spectroscopic Evidence of Superconductivity Pairing at $83 \mathrm{~K}$ in Single-Layer FeSe/SrTiO3 Films, Nat. Commun. 12, 2840 (2021). 